\title{
Initial boundary value problems for the three-dimensional compressible elastic Navier-Stokes-Poisson equations
}

https://doi.org/10.1515/anona-2020-0184

Received February 28, 2021; accepted April 5, 2021.

Abstract: We study the initial-boundary value problems of the three-dimensional compressible elastic NavierStokes-Poisson equations under the Dirichlet or Neumann boundary condition for the electrostatic potential. The unique global solution near a constant equilibrium state in $H^{2}$ space is obtained. Moreover, we prove that the solution decays to the equilibrium state at an exponential rate as time tends to infinity. This is the first result for the three-dimensional elastic Navier-Stokes-Poisson equations under various boundary conditions for the electrostatic potential.

Keywords: Elastic Navier-Stokes-Poisson equations; Initial-boundary value problems; Global solution; Exponential decay

MSC: 76A10; 35Q35; 35G61

\section{Introduction}

As is known to all, solids have elastic behaviors so that the deformations happened in solids recover once the stress field is removed, however, fluids possess the viscous property which plays a role of internal frictions to dissipate the kinetic energy of fluids. Viscoelastic fluids we concern here lie between elastic solids and viscous fluids, which flow like fluids and demonstrate elastic behaviors. This kind of fluids often have more complex microstructures than usual fluids (eg. water and air). A typical example is a polymer containing a large number of long-chain molecules. When these long-chain molecules are stretched out due to flow, an elastic stress will appear to hinder the stretched deformations. In general, there are three kinds of stresses in viscoelastic fluids: the hydrostatic pressure $P$, the viscous stress $\mathbb{T}_{v}$ and the elastic stress $\mathbb{T}_{e}$. So the total stress tensor $\mathbb{T}^{\text {total }}$ can be expressed as

$$
\mathbb{T}^{\text {total }}=-P \mathbb{I}+\mathbb{T}_{v}+\mathbb{T}_{e},
$$

where $\mathbb{I}$ is the identity matrix. The viscous stress $\mathbb{T}_{v}$ depends on the rate of strain, however, the elastic stress $\mathbb{T}_{e}$ can be only determined by the deformation gradient. The combined stress governs the motion of viscoelastic fluids. Many viscoelastic fluids, from a microscopic point of view, are composed of a great many of charged particles, and at the macro level, they behave as the electrical conducting fluid motion. About more backgrounds, the readers can refer to [23, 32, 42]. In this paper, we focus on the dynamics of the compressible viscoelastic electrical conducting fluids. The readers will notice that there are many kinds of viscoelastic fluid models, in which the elastic stress obeys a constitutive law of differential or integral type, cf. [18, 21, 42].

\footnotetext{
Yong Wang, South China Research Center for Applied Mathematics and Interdisciplinary Studies, School of Mathematical Sciences, South China Normal University, Guangzhou, 510631, China, E-mail: wangyongxmu@163.com

*Corresponding Author: Wenpei Wu, Academy of Mathematics and Systems Science, Chinese Academy of Sciences, Beijing, 100190, China and School of Mathematical Sciences, Xiamen University, Xiamen, 361005, China,

E-mail: wenpeiwu16@163.com
} 
However, we shall be devoted to studying the third type, i.e., the elastic stress depends only on the current deformation gradient.

To derive a PDE's model to describe the dynamics of the compressible viscoelastic electrical conducting fluids, we start from the following energy dissipation law: $(x, t) \in \Omega \times \mathbb{R}^{+}\left(\Omega \subset \mathbb{R}^{3}\right)$,

$$
\begin{aligned}
\frac{d}{d t} E^{\text {total }} & :=\frac{d}{d t} \int_{\Omega}\left[\frac{1}{2} \rho|u|^{2}+\omega(\rho)+\frac{1}{2} c^{2} \rho|\mathbb{F}|^{2}+\frac{1}{2}|\nabla \phi|^{2}\right] d x \\
& =-\int_{\Omega}\left[\mu|\nabla u|^{2}+(\mu+\lambda)|\nabla \cdot u|^{2}\right] d x:=-\triangle
\end{aligned}
$$

where $\rho, u, \mathbb{F}, \phi$ denote the density, the velocity, the deformation gradient and the electrostatic potential, respectively. Here the total energy $E^{\text {total }}$ per unit volume includes the kinetic energy $\frac{1}{2} \rho|u|^{2}$, the internal energy $\omega(\rho)$ depending on the fluid density $\rho$, the elastic potential energy $\frac{1}{2} c^{2} \rho|\mathbb{F}|^{2}$ and the electric energy $\frac{1}{2}|\nabla \phi|^{2}$. The above constant $c>0$ represents the speed of elastic wave propagation. For simplicity, we have assumed:

(1) The electric energy is only generated by the electrostatic field $E_{s}:=-\nabla \phi$;

(2) The dissipation is only caused by the fluid viscosity;

(3) The viscosity is chosen to follow Newton's law of viscosity.

Here the assumptions (2) and (3) imply that the dissipation (entropy production) $\triangle$ per unit volume is equal to $\mu|\nabla u|^{2}+(\mu+\lambda)|\nabla \cdot u|^{2}$, where $\mu$ and $\lambda$ represent the shear and bulk viscosity coefficients, respectively. The key point of the modeling is to derive the motion equation. The total force is the combination of the pressure, the viscous stress, the elastic stress and the Coulomb force due to the electrostatic field. It is natural to use the momentum conservation law to write down a PDE:

$$
(\rho u)_{t}+\nabla \cdot(\rho u \otimes u)=\rho \nabla \phi+\nabla \cdot \mathbb{T}^{\text {total }},
$$

where $\otimes$ denotes the tensor product. Next one should figure out what is the total stress $\mathbb{T}^{\text {total }}$ and how does it depend on other unknowns. The problem is tough since the total stress is relatively complicated for viscoelastic fluids. However, using an energetic variational approach based on the energy dissipation law (1.1), the authors can derive the motion equation [46]:

$$
(\rho u)_{t}+\nabla \cdot(\rho u \otimes u)=\rho \nabla \phi-\nabla P+\mu \Delta u+(\mu+\lambda) \nabla \nabla \cdot u+c^{2} \nabla \cdot\left(\rho \mathbb{F}^{T}\right),
$$

from where one can know that

$$
\mathbb{T}_{v}=\lambda \operatorname{div} u \mathbb{I}+2 \mu \mathbb{D}(u), \quad \mathbb{T}_{e}=c^{2} \rho \mathbb{F F}^{T}, \quad \mathbb{D}(u)=\frac{1}{2}\left[\nabla u+(\nabla u)^{T}\right]
$$

and the pressure $P$ is determined by an ODE:

$$
P=P(\rho)=\rho \omega^{\prime}(\rho)-\omega(\rho) .
$$

In the above, the superscript $T$ denotes the transpose of the matrix. Coupled (1.2) with the continuity equation for $\rho$, the transport equations for $\mathbb{F}$ and the Poisson's equation for $\phi$, one can obtain the following closed system [46]:

$$
\left\{\begin{array}{l}
\rho_{t}+\nabla \cdot(\rho u)=0, \\
(\rho u)_{t}+\nabla \cdot(\rho u \otimes u)+\nabla P(\rho)=\mu \Delta u+(\mu+\lambda) \nabla \nabla \cdot u+c^{2} \nabla \cdot\left(\rho \mathbb{F}^{T}\right)+\rho \nabla \phi, \\
\mathbb{F}_{t}+u \cdot \nabla \mathbb{F}=\nabla u \mathbb{F}, \\
\Delta \phi=\rho-\bar{\rho}, \quad(x, t) \in \Omega \times \mathbb{R}^{+}, \quad \Omega \subset \mathbb{R}^{3} .
\end{array}\right.
$$

We say that such a system (1.4) is thermodynamically consistent and the constitutive relations for stresses in (1.4) satisfy the principle of material frame indifference. To illustrate those two points, we simply recall the 
derivation of the system (1.4). Based on the first and second laws of thermodynamics, by assuming that the flow is isothermal and not affected by external forces, we can deduce the formal energy dissipation law as follows. It holds that

$$
\left.\begin{array}{l}
\text { 1st law: } \frac{d(\mathcal{K}+\mathcal{J})}{d t}=\frac{d \mathcal{W}}{d t}+\frac{d Q}{d t} \\
\text { 2nd law: } \theta \frac{d \mathcal{S}}{d t}=\frac{d Q}{d t}+\triangle
\end{array}\right\} \stackrel{\theta=\bar{\theta}}{=} \frac{d}{\mathcal{W}=0} E^{\text {total }}=-\triangle,
$$

where the symbols $\mathcal{K}, \mathcal{J}, \mathcal{W}, Q, \mathcal{S}$ and $\triangle$ are the kinetic energy, the internal energy, the work of external forces, the heat, the entropy and the entropy production (or dissipation), respectively. Assume that the absolute temperature $\theta$ values a positive constant $\bar{\theta}$. So the total energy $E^{\text {total }}=\mathcal{K}+\mathcal{J}-\bar{\theta} \mathcal{S}$. There is no external force which means that $\mathcal{W}=0$. By setting the total energy $E^{\text {total }}$ and the dissipation $\triangle$, like (1.1), and applying the energetic variational approach as done in [46], one can derive the closed system (1.4). Reversely, by multiplying Eqs. $(1.4)_{1},(1.4)_{2}$ and $(1.4)_{3}$ by $\omega^{\prime}(\rho)-\phi, u$ and $c^{2} \rho \mathbb{F}$, respectively, summing them up and then integrating over $\Omega$, where integrating by parts under the conditions of $\left.u\right|_{\partial \Omega}=0$ and $\left.\phi\right|_{\partial \Omega}=0$ or $\left.\nabla \phi \cdot v\right|_{\partial \Omega}=0(v$ unit outward normal to $\partial \Omega$ ), one can back to the energy dissipation law (1.1). In this sense, the system (1.4) is thermodynamically consistent. Moreover, all the constitutive relations for stresses satisfy the principle of material objectivity or frame indifference $[1,42,49]$. This principle requires that the stress results only from deformations and is unaffected (excepted for orientation) by rigid rotations of the body. For example, this restriction requires that the elastic stress $\widetilde{\mathbb{T}}$ satisfies

$$
\mathbb{Q} \widetilde{\mathbb{T}}(\mathbb{F}) \mathbb{Q}^{T}=\widetilde{\mathbb{T}}(\mathbb{Q F}), \quad \forall \mathbb{Q} \in \mathrm{SO}(3),
$$

where $\mathbb{Q}: \mathbb{R} \rightarrow \mathbb{R}^{3 \times 3}$ and $\mathrm{SO}(3)$ is the $3 \times 3$ special orthogonal group (means that $\mathbb{Q Q}^{T}=\mathbb{I}$ and $\operatorname{det} \mathbb{Q}=1$ ). It is easy to verify that $\mathbb{T}_{e}=c^{2} \rho \mathbb{F F}^{T}$ in (1.3) satisfies the relation (1.5). In [46], Tan and the authors of this paper studied the global well-posedness of the three-dimensional Cauchy problem of (1.4) $\left(\Omega=\mathbb{R}^{3}\right)$. As a work of continuity, this paper will deal with the corresponding initial-boundary value problems in dimension three, in which the electrostatic potential shall be assigned to Dirichlet or Neumann boundary conditions.

To be precise, we will study the initial-boundary value problems of the compressible elastic NavierStokes-Poisson equations:

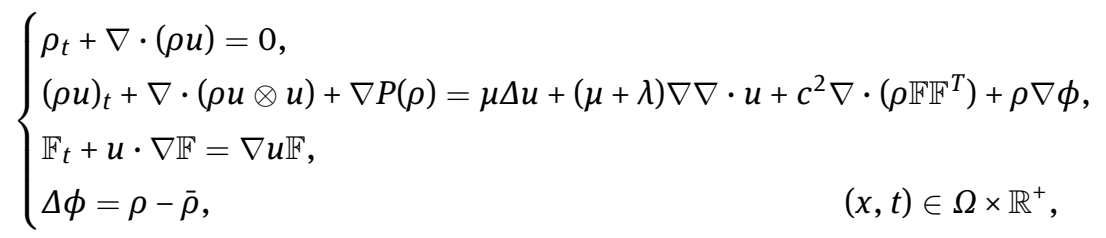

with the initial and boundary conditions

$$
\begin{cases}\left.(\rho, u, \mathbb{F})(x, t)\right|_{t=0}=\left(\rho_{0}, u_{0}, \mathbb{F}_{0}\right)(x), & x \in \Omega, \\ \left.u\right|_{\partial \Omega}=0, & t>0\end{cases}
$$

and

$$
\left.\phi\right|_{\partial \Omega}=0 \text { or }\left.\nabla \phi \cdot v\right|_{\partial \Omega}=0, \quad t>0 .
$$

Here, $\Omega \subset \mathbb{R}^{3}$ is a bounded region and $v$ is the unit outward normal to $\partial \Omega$. The unknown variables $\rho=$ $\rho(x, t)>0, u=u(x, t) \in \mathbb{R}^{3}, \mathbb{F}=\mathbb{F}(x, t) \in \mathbb{M}^{3 \times 3}$ (the set of $3 \times 3$ matrices with positive determinants) denote the density, the velocity and the deformation gradient of viscoelastic electrical conducting fluids, respectively. The electrostatic potential $\phi=\phi(x, t)$ is coupled with the density through the Poisson equation. The pressure $P=P(\rho)$ is a smooth function satisfying $P^{\prime}(\rho)>0$ for $\rho>0$. Two constant viscosity coefficients $\mu$ and $\lambda$ satisfy the usual physical constraints $\mu>0$ and $3 \lambda+2 \mu \geqslant 0$. In the motion of fluids, we use $\bar{\rho}>0$ to model a constant background charge distribution. Without loss of generality, we assume

$$
\bar{\rho}=\frac{1}{|\Omega|} \int_{\Omega} \rho d x=c=1 .
$$


Here we make an emphasis on physical meanings of the above boundary conditions. The vanishing velocity $u$ on the boundary can be well understood as the non-slip boundary condition due to the fluid viscosity. The homogeneous Dirichlet-type boundary condition for the electrostatic potential $\phi$ implies that the boundary is grounded. In addition, the homogeneous Neumann-type boundary condition means that the boundary is well-insulated.

Now we review the history on the non-conducting viscoelastic system corresponding to the equations (1.6):

$$
\left\{\begin{array}{l}
\rho_{t}+\nabla \cdot(\rho u)=0 \\
(\rho u)_{t}+\nabla \cdot(\rho u \otimes u)+\nabla P(\rho)=\mu \Delta u+(\mu+\lambda) \nabla \nabla \cdot u+c^{2} \nabla \cdot\left(\rho \mathbb{F F}^{T}\right) \\
\mathbb{F}_{t}+u \cdot \nabla \mathbb{F}=\nabla u \mathbb{F} .
\end{array}\right.
$$

About the Cauchy problem of the system (1.9), Hu and Wang [13] proved the local existence and uniqueness of the strong solution with large initial data. Later, $\mathrm{Hu}$ and $\mathrm{Wu}$ [17] generalized the local unique strong solution to the global one in the framework of Matsumura and Nishida [34, 35] and got the optimal time-decay rates of lower-order spatial derivatives via semigroup methods developed in $[9,39]$ under the condition that the initial data belong to $L^{1}\left(\mathbb{R}^{3}\right)$. Based on the same $L^{1}$ assumption for the initial data, the optimal time-decay rates of higher-order spatial derivatives were obtained by Li et al. [29] who used the Fourier splitting method (cf. $[43,44]$ ). Under the weaker assumption in the sense that one replaces $L^{1}\left(\mathbb{R}^{3}\right)$ with the homogeneous Besov space $\dot{B}_{2, \infty}^{-3 / 2}\left(\mathbb{R}^{3}\right)$ due to $L^{1} \subset \dot{B}_{2, \infty}^{-3 / 2}$, Wu et al. [53] obtained the optimal time-decay rates of arbitrary spatial derivatives, where a pure energy method introduced in [7] was used. Besides, some related results of the system (1.9) can be seen in [11, 14, 15, 41] and the references therein. As for the initial-boundary value problem of the system (1.9), Qian [40] proved that the global-in-time solution exists uniquely near the equilibrium state in $H^{2}\left(\mathbb{R}^{3}\right)$ space and then Chen and Wu [3] showed the exponential decay rates. Hu and Wang [16] also obtained the unique global solution in a lower regularity space, say $W^{1, q}\left(\mathbb{R}^{3}\right)(q>3)$. When the density is constant, the system (1.9) will become the incompressible viscoelastic fluid equations. For the incompressible problems, the readers can refer to $[4,12,19,20,24-27,30,31,55]$ and the references cited therein.

Without considering the elasticity, the system (1.6) becomes the compressible Navier-Stokes-Poisson equations:

$$
\left\{\begin{array}{l}
\rho_{t}+\nabla \cdot(\rho u)=0, \\
(\rho u)_{t}+\nabla \cdot(\rho u \otimes u)+\nabla P(\rho)=\mu \Delta u+(\mu+\lambda) \nabla \nabla \cdot u+\rho \nabla \phi, \\
\Delta \phi=\rho-\bar{\rho} .
\end{array}\right.
$$

For the Cauchy problem of the system (1.10), there are a lot of results, cf. [2, 8, 10, 28, 47, 50-52, 54] and the references therein. In a sense, the initial-boundary value problem of the Navier-Stokes-Poisson system is more difficult than its Cauchy problem. For initial-boundary value problems, it is necessary to estimate the boundary integral terms involving higher-order derivatives, however, these terms are often out of control due to the loss of the boundary information of derivatives. This means that one cannot obtain higher-order energy estimates by the usual energy methods. An effective method was introduced by Matsumura and Nishida [36, 37] to deal with the initial-boundary value problems of the Navier-Stokes equations. However, the Poisson term $\rho \nabla \phi$ brings essential difficulties when considering the initial-boundary value problem of the system (1.10). The reason is that one cannot obtain the dissipation estimates of the electric field $-\nabla \phi$ whenever the boundary condition for the electrostatic potential is Dirichlet-type or Neumann-type or other else. Given this point, it is not like the Cauchy problem, where the electric field enhances the decay of the density if adding some additional restrictions to the initial electric field, cf. [51]. However, we found a very interesting phenomenon. Under the influence of the elasticity, we can obtain the effective dissipation estimates of $\nabla \phi$ so that the Dirichlet or Neumann boundary value problems can be solved. Very recently, we learn that the Neumann problem for the system (1.10) has been solved by Liu and Zhong [33], while, the Dirichlet problem is still open.

The novelty of this paper mainly includes two points. One is to develop the effects of elasticity variables (not the deformation gradient $\mathbb{F}$ but the deformation $\varphi=X(x, t)-x$ introduced in Section 2). Given two important relations (2.4) and (2.6), it suffices to solve the equations (2.3) about $\varphi$ and then one can immediately 
obtain $(\rho, \mathbb{F})$. Moreover, both the relation (2.6) and the Poisson equation (1.6) ${ }_{4}$ provide an effective connection between the electrostatic potential $\phi$ and the deformation $\varphi$, say, $\Delta \phi=\nabla \cdot \varphi+O\left(\left|\nabla \varphi^{2}\right|\right)$, which plays an important role in deriving the estimates for $\nabla \phi$ as stated in Lemma 3.3. The other point lies in that we can uniformly deal with two kinds of important boundary-value problems: Dirichlet type and Neumann type. Our results are relatively non-trivial since different difficulties will appear under different boundary conditions. Here we make a series of delicate energy estimates (see Lemmas 3.1-3.10), which are all applicable for these boundary conditions. Our treatment is clean and effective, which shall shed light on similar boundary value problems of other models.

Notation. Throughout this paper, we use $a \lesssim b$ if $a \leqslant C b$ for a universal constant $C>0$. The relation $a \sim b$ means that $a \lesssim b$ and $b \lesssim a$. We denote the gradient operator $\nabla=\partial_{x}=\left(\partial_{x_{1}}, \partial_{x_{2}}, \partial_{x_{3}}\right)^{T}$ and $\nabla_{j}:=\partial_{x_{j}}$ $(j=1,2,3)$. We denote the Frobenius inner product of two matrices $\mathbb{A}, \mathbb{B} \in \mathbb{R}^{3 \times 3}$ by $\mathbb{A}: \mathbb{B}:=\sum_{i, j=1}^{3} \mathbb{A}^{i j} \mathbb{B}^{i j}$. Particularly, $|\mathbb{A}|^{2}=\mathbb{A}: \mathbb{A}$. The usual Sobolev spaces are denoted by $H^{k}=W^{k, 2}(k=1,2, \ldots)$ equipped with the norm $\|\cdot\|_{H^{k}}$. The usual Lebesgue spaces are denoted by $L^{p}(1 \leqslant p \leqslant \infty)$ equipped with the norm $\|\cdot\|_{L^{p}}$. We always write $\|\cdot\|=\|\cdot\|_{L^{2}}$ for brevity. The spaces involving time $L^{p}([0, T] ; Z)$ denote all the measurable functions $f:[0, T] \rightarrow Z$ with the norm $\|f\|_{L^{p}([0, T] ; Z)}:=\left(\int_{0}^{T}\|f(t)\|_{Z}^{p} d t\right)^{1 / p}<\infty$ for $1 \leqslant p<\infty$. The spaces involving time $\mathfrak{C}([0, T] ; Z)$ denote all the continuous functions $f:[0, T] \rightarrow Z$ with the norm $\|f\|_{\mathcal{C}([0, T] ; Z)}:=$ $\max _{0 \leqslant t \leqslant T}\|f(t)\|_{Z}<\infty$.

This paper is organized as follows. We make a reformulation for the original problem (1.6)-(1.8) and list the main results in Section 2. In Section 3, we establish the delicate energy estimates of solutions for the linearized system. In Section 4, we complete the proof of Theorem 2.1 by deducing the a priori estimates from the energy estimates in Section 3. In the appendix, we list some auxiliary lemmas needed in the previous sections.

\section{Main results}

In this section, we first make a reformulation of the original problem and then state the main results on the existence, uniqueness and large-time behaviors (exponential decay rates) of solutions.

\subsection{Reformulation}

We denote $x$ as the current spatial (Eulerian) coordinate and $X$ as the material (Lagrangian) coordinate for fluid particles. These two coordinates are connected by the flow map $x(X, t)$ defined by the following system of ordinary differential equations:

$$
\left\{\begin{array}{l}
\frac{d x(X, t)}{d t}=u(x(X, t), t), \quad t>0, \\
x(X, 0)=X
\end{array}\right.
$$

where $u(x(X, t), t)$ is a given velocity field. Then the deformation gradient $\widetilde{F}$ is defined as

$$
\widetilde{\mathbb{F}}(X, t)=\frac{\partial x(X, t)}{\partial X} .
$$

When considering it in the Eulerian coordinate, the deformation gradient $\mathbb{F}(x, t)$ will be defined as

$$
\mathbb{F}(x(X, t), t)=\widetilde{\mathbb{F}}(X, t) .
$$

By the chain rule, we easily prove that $\mathbb{F}(x, t)$ satisfies the following transport equations:

$$
\mathbb{F}_{t}+u \cdot \nabla \mathbb{F}=\nabla u \mathbb{F} .
$$


Next, we will reformulate the system (1.6). We introduce the inverse of $\mathbb{F}$ by

$$
\mathbb{E}:=\frac{\partial X}{\partial x}=\mathbb{F}^{-1},
$$

where $X=X(x, t)$ is the inverse mapping of $x(X, t)$. We define the quantity

$$
\mathbb{K}:=\mathbb{E}-\mathbb{I},
$$

which was first introduced by Sideris and Thomases [45]. Note that the matrix $\mathbb{K}=\left(\mathbb{K}^{i j}\right)$ is curl free (cf. [31]), so there exists a vector valued function $\varphi=\left(\varphi^{1}, \varphi^{2}, \varphi^{3}\right)^{T}$ such that $\left(\mathbb{K}^{i 1}, \mathbb{K}^{i 2}, \mathbb{K}^{i 3}\right)^{T}=\nabla \varphi^{i}(i=1,2,3)$. In fact, the function $\varphi$ can be chosen to be $\varphi(x, t)=X(x, t)-x$, which implies

$$
\varphi_{t}+u \cdot \nabla \varphi+u=0 .
$$

Due to $\left.u\right|_{\partial \Omega}=0$, it holds that $\left.\varphi\right|_{\partial \Omega}=0$. By (2.1)-(2.2) and the Taylor's expansion, we have

$$
\mathbb{F}=(\mathbb{I}+\mathbb{K})^{-1}=\sum_{i=0}^{\infty}(-1)^{i} \mathbb{K}^{i}=\mathbb{I}-\mathbb{K}+O\left(|\mathbb{K}|^{2}\right)=\mathbb{I}-\nabla \varphi+O\left(|\nabla \varphi|^{2}\right),
$$

where the absolute convergence of the matrix series is insured due to $\|\nabla \varphi\|_{H^{2}} \ll 1$ in later discussions. From the fact that $\nabla \cdot\left(\rho \mathbb{F}^{T}\right)(t)=\nabla_{j}\left(\rho \mathbb{F}^{j k}\right)=0$ for all $t \geqslant 0$ stated in Lemma A.3, we shall deduce that the $i$-th component of the vector $\frac{1}{\rho} \nabla \cdot\left(\rho \mathbb{F F}^{T}\right)$ as follows:

$$
\begin{aligned}
\frac{1}{\rho} \nabla_{j}\left(\rho \mathbb{F}^{i k} \mathbb{F}^{j k}\right) & =\frac{1}{\rho} \mathbb{F}^{i k} \nabla_{j}\left(\rho \mathbb{F}^{j k}\right)+\mathbb{F}^{j k} \nabla_{j} \mathbb{F}^{i k}=\mathbb{F}^{j k} \nabla_{j} \mathbb{F}^{i k} \\
& =\left(\delta^{j k}-\mathbb{K}^{j k}+O\left(|\mathbb{K}|^{2}\right)\right) \nabla_{j}\left(\delta^{i k}-\mathbb{K}^{i k}+O\left(|\mathbb{K}|^{2}\right)\right) \\
& =-\nabla_{k} \mathbb{K}^{i k}+O(|\mathbb{K}|) \nabla O(|\mathbb{K}|) \\
& =-\Delta \varphi^{i}+O(|\nabla \varphi|) \nabla O(|\nabla \varphi|) .
\end{aligned}
$$

Through the fact $\rho \operatorname{det} \mathbb{F}=1$ for all $t \geqslant 0$ in Lemma A.3 and the determinant expansion theorem, we have

$$
\rho=\operatorname{det} \mathbb{F}^{-1}=\operatorname{det}(\mathbb{I}+\nabla \varphi)=1+\nabla \cdot \varphi+\frac{1}{2}\left\{(\nabla \cdot \varphi)^{2}-\operatorname{tr}\left[(\nabla \varphi)^{2}\right]\right\}+\operatorname{det}(\nabla \varphi),
$$

which implies

$$
\rho-1=\nabla \cdot \varphi+O\left(|\nabla \varphi|^{2}\right)
$$

Next, we define the material derivative

$$
\frac{D}{D t}:=\partial_{t}+u \cdot \nabla
$$

Applying the divergence $\nabla \cdot$ to both sides of (2.3), we have

$$
\frac{D(\nabla \cdot \varphi)}{D t}+\nabla \cdot u=-\operatorname{tr}(\nabla u \nabla \varphi)=-(\nabla u)^{T}: \nabla \varphi .
$$

For simplicity, we take $P^{\prime}(1)=1$. Thus, using (2.3)-(2.6), we can rewrite (1.6) into the linearized form as

$$
\left\{\begin{array}{l}
L_{1}:=u_{t}-\mu \Delta u-(\mu+\lambda) \nabla(\nabla \cdot u)+\Delta \varphi+\nabla(\nabla \cdot \varphi)-\nabla \phi=R_{1}, \\
L_{2}:=\varphi_{t}+u=R_{2} \\
\Delta \phi=\nabla \cdot \varphi+O\left(|\nabla \varphi|^{2}\right)
\end{array}\right.
$$

which is subject to the initial and boundary conditions

$$
\begin{cases}\left.(u, \varphi)(x, t)\right|_{t=0}=\left(u_{0}, \varphi_{0}\right)(x), & x \in \Omega \\ \left.u\right|_{\partial \Omega}=\left.\varphi\right|_{\partial \Omega}=0, & t>0 \\ \left.\phi\right|_{\partial \Omega}=0 \text { or }\left.\nabla \phi \cdot v\right|_{\partial \Omega}=0, & t>0\end{cases}
$$


In the above, we define

$$
\left\{\begin{aligned}
R_{1}: & =-u \cdot \nabla u-\left(1-\frac{1}{\rho}\right)[\mu \Delta u+(\mu+\lambda) \nabla(\nabla \cdot u)] \\
& -\left(\frac{P^{\prime}(\rho)}{\rho}-1\right) \nabla\left[\nabla \cdot \varphi+O\left(|\nabla \varphi|^{2}\right)\right]+O(|\nabla \varphi|) \nabla O(|\nabla \varphi|), \\
R_{2}: & =-u \cdot \nabla \varphi .
\end{aligned}\right.
$$

Since $\|\nabla \varphi\|_{H^{2}} \ll 1$ in the following a priori estimates, by (2.6), we have

$$
\frac{1}{2} \leqslant \rho \leqslant \frac{3}{2}
$$

By the Taylor's expansion and (2.6), we get

$$
1-\frac{1}{\rho}, \frac{P^{\prime}(\rho)}{\rho}-1 \sim \rho-1=\nabla \cdot \varphi+O\left(|\nabla \varphi|^{2}\right),
$$

which infers

$$
R_{1} \approx u \cdot \nabla u+\nabla \varphi \nabla^{2} u+\nabla \varphi \nabla^{2} \varphi+\text { cubic terms. }
$$

\subsection{Main results}

Our main results are stated in the following: the global existence, uniqueness and exponential decay of solutions.

Theorem 2.1. Let $\Omega \subset \mathbb{R}^{3}$ be a bounded domain with $\partial \Omega \in \mathcal{C}^{3}$. Assume that $\left(\rho_{0}-1, u_{0}, \mathbb{F}_{0}-\mathbb{I}\right) \in H^{2}(\Omega)$ satisfying

$$
\left\{\begin{array}{l}
\nabla \cdot\left(\rho_{0} \mathbb{F}_{0}^{T}\right)=0, \quad \rho_{0} \operatorname{det} \mathbb{F}_{0}=1 \\
\mathbb{F}_{0}^{l k} \nabla_{l} \mathbb{F}_{0}^{i j}=\mathbb{F}_{0}^{l j} \nabla_{l} \mathbb{F}_{0}^{i k}, \\
\mathbb{F}_{0}=\left(\mathbb{I}+\nabla \varphi_{0}\right)^{-1} \quad \text { for some } \varphi_{0} \in H^{3} \cap H_{0}^{1} \\
\int_{\Omega}\left(\rho_{0}-1\right) d x=0
\end{array}\right.
$$

Then there exists a suitably small constant $\delta_{0}>0$ such that if

$$
\left\|\left(\rho_{0}-1, u_{0}, \mathbb{F}_{0}-\mathbb{I}\right)\right\|_{H^{2}}<\delta_{0},
$$

then the initial-boundary value problem (1.6)-(1.8) admits a unique global solution $(\rho, u, \mathbb{F}, \nabla \phi)$ satisfying

$$
\left\{\begin{array}{l}
\rho \in \mathcal{C}\left([0,+\infty) ; H^{2}(\Omega)\right), \quad \rho_{t} \in \mathcal{C}\left([0,+\infty) ; H^{1}(\Omega)\right), \\
u \in \mathcal{C}\left([0,+\infty) ; H^{2}(\Omega) \cap H_{0}^{1}(\Omega)\right) \cap L^{2}\left([0,+\infty) ; H^{3}(\Omega)\right), \\
u_{t} \in \mathcal{C}\left([0,+\infty) ; L^{2}(\Omega)\right) \cap L^{2}\left([0,+\infty) ; H_{0}^{1}(\Omega)\right), \\
\mathbb{F} \in \mathcal{C}\left([0,+\infty) ; H^{2}(\Omega)\right), \quad \mathbb{F}_{t} \in \mathcal{C}\left([0,+\infty) ; H^{1}(\Omega)\right), \\
\nabla \phi \in \mathcal{C}\left([0,+\infty) ; H^{3}(\Omega)\right), \quad \nabla \phi_{t} \in \mathcal{C}\left([0,+\infty) ; H^{2}(\Omega)\right) .
\end{array}\right.
$$

Furthermore, there exists a constant $\alpha>0$ such that for all $t \geqslant 0$,

$$
\|(\rho-1, u, \mathbb{F}-\mathbb{I}, \nabla \phi)(t)\|_{H^{2}}+\left\|\left(\rho_{t}, u_{t}, \mathbb{F}_{t}, \nabla \phi_{t}\right)(t)\right\| \leqslant C_{0} e^{-\alpha t},
$$

where $C_{0}>0$ depends only on the initial data.

Finally, we give some remarks. 
Remark 2.1. Since it is hard to impose the boundary conditions for the deformation gradient $\mathbb{F}$, it turns to introduce a vector-valued function $\varphi$, which satisfies the transport equation (2.3) and the reasonable boundary condition $\left.\varphi\right|_{\partial \Omega}=0$ by $\left.u\right|_{\partial \Omega}=0$.

Remark 2.2. Given the relations (2.4) and (2.6), we can drop the equations for $\rho$ and $\mathbb{F}$ by solving the system (2.8) only involving three unknowns $u, \varphi$ and $\phi$. This greatly simplifies the proof processes, see Section 3.

Remark 2.3. In this paper, we try to pursue global-in-time solutions with minimal regularity. In fact, the global $H^{2}$-regularity solution obtained in Theorem 2.1 can become more regular if improving the smoothness of the boundary $\partial \Omega$ and the initial data.

Remark 2.4. We easily deduce the regularity of the electrostatic potential $\phi$ from Theorem 2.1:

$$
\begin{cases}\phi \in \mathcal{C}\left([0,+\infty) ; H^{4}(\Omega)\right), \quad \phi_{t} \in \mathcal{C}\left([0,+\infty) ; H^{3}(\Omega)\right), & \text { if }\left.\phi\right|_{\partial \Omega}=0, \\ \phi-\phi_{\Omega} \in \mathcal{C}\left([0,+\infty) ; H^{4}(\Omega)\right), \quad \phi_{t}-\phi_{t \Omega} \in \mathcal{C}\left([0,+\infty) ; H^{3}(\Omega)\right), & \text { if }\left.\nabla \phi \cdot v\right|_{\partial \Omega}=0, f_{\Omega}:=\frac{1}{|\Omega|} \int_{\Omega} f d x\end{cases}
$$

by Lemma A.1 (Poincarés inequality).

\section{Energy estimates}

For completeness, we first give the local existence and uniqueness of the strong solution of the problem (1.6)(1.8) and omit its proof, cf. [22].

Proposition 3.1. Let $\Omega \subset \mathbb{R}^{3}$ be a bounded domain with $\partial \Omega \in \mathcal{C}^{3}$. Assume that $\left(\rho_{0}-1, u_{0}, \mathbb{F}_{0}-\mathbb{I}\right) \in H^{2}(\Omega)$ satisfying $\inf _{x \in \bar{\Omega}}\left\{\rho_{0}(x)\right\}>0$ and (2.10). Then there exists a constant $T>0$ such that the initial-boundary value problem (1.6)-(1.8) has a unique solution $(\rho, u, \mathbb{F}, \nabla \phi)(t)$ such that

$$
\left\{\begin{array}{l}
\rho \in \mathcal{C}\left([0, T] ; H^{2}(\Omega)\right), \quad \rho_{t} \in \mathcal{C}\left([0, T] ; H^{1}(\Omega)\right), \\
u \in \mathcal{C}\left([0, T] ; H^{2}(\Omega) \cap H_{0}^{1}(\Omega)\right) \cap L^{2}\left([0, T] ; H^{3}(\Omega)\right), \\
u_{t} \in \mathcal{C}\left([0, T] ; L^{2}(\Omega)\right) \cap L^{2}\left([0, T] ; H_{0}^{1}(\Omega)\right), \\
\mathbb{F} \in \mathcal{C}\left([0, T] ; H^{2}(\Omega)\right), \quad \mathbb{F}_{t} \in \mathcal{C}\left([0, T] ; H^{1}(\Omega)\right), \\
\nabla \phi \in \mathcal{C}\left([0, T] ; H^{3}(\Omega)\right), \quad \nabla \phi_{t} \in \mathcal{C}\left([0, T] ; H^{2}(\Omega)\right)
\end{array}\right.
$$

and

$$
\left\{\begin{array}{l}
\inf _{(x, t) \in \bar{\Omega} \times[0, T]} \rho(x, t) \geqslant \frac{1}{2}+\frac{1}{2} \inf _{x \in \bar{\Omega}}\left\{\rho_{0}(x)\right\}>0, \\
\sup _{0 \leqslant t \leqslant T}\|(\rho-1, u, \mathbb{F}-\mathbb{I}, \nabla \phi)(t)\|_{H^{2}} \leqslant C_{1}\left\|\left(\rho_{0}-1, u_{0}, \mathbb{F}_{0}-\mathbb{I}\right)\right\|_{H^{2}},
\end{array}\right.
$$

where $C_{1}>1$ is some fixed constant.

To obtain the global-in-time solution of the problem (1.6)-(1.8), we shall make many efforts to derive the a priori estimates. Note that the relations (2.4) and (2.6)

$$
\left\{\begin{array}{l}
\mathbb{F}-\mathbb{I}=-\nabla \varphi+O\left(|\nabla \varphi|^{2}\right) \\
\rho-1=\nabla \cdot \varphi+O\left(|\nabla \varphi|^{2}\right)
\end{array}\right.
$$

It suffices to derive the energy estimates of the solution $(u, \varphi, \nabla \phi)$ to the linearized system (2.8).

We assume that for some sufficiently small $\epsilon>0$ and some $T>0$,

$$
\sup _{0 \leqslant t \leqslant T}\|(\rho-1, u, \mathbb{F}-\mathbb{I})(t)\|_{H^{2}}<\epsilon,
$$


which implies

$$
\sup _{0 \leqslant t \leqslant T}\|(u, \nabla \varphi, \nabla \phi)(t)\|_{H^{2}}<\epsilon .
$$

We first establish the dissipation estimate for $\nabla u$.

Lemma 3.1. It holds that

$$
\frac{d}{d t}\|(u, \nabla \cdot \varphi, \nabla \varphi, \nabla \phi)\|^{2}+\|\nabla u\|^{2} \lesssim \epsilon\left\|\left(\nabla \varphi, \nabla^{2} \varphi\right)\right\|^{2} .
$$

Proof. Integrating the resulting identity $u \cdot\left(L_{1}-R_{1}\right)-\Delta \varphi \cdot\left(L_{2}-R_{2}\right)=0$ over $\Omega$, integrating by parts and using $\left.u\right|_{\partial \Omega}=\left.\varphi\right|_{\partial \Omega}=0$ and (1.8), we obtain

$$
\begin{aligned}
& \frac{1}{2} \frac{d}{d t} \int_{\Omega}\left(|u|^{2}+|\nabla \cdot \varphi|^{2}+|\nabla \varphi|^{2}+|\nabla \phi|^{2}\right) d x+\int_{\Omega}\left(\mu|\nabla u|^{2}+(\mu+\lambda)|\nabla \cdot u|^{2}\right) d x \\
& =\int_{\Omega}\left[R_{1} \cdot u-R_{2} \cdot \Delta \varphi-\nabla \cdot(u \cdot \nabla \varphi)(\nabla \cdot \varphi)-(u \cdot \nabla \varphi) \cdot \nabla \phi\right] d x \\
& \quad- \begin{cases}\int_{\Omega} O\left(|\nabla \varphi|^{2}\right)_{t} \phi d x, & \text { if }\left.\phi\right|_{\partial \Omega}=0, \\
\int_{\Omega} O\left(|\nabla \varphi|^{2}\right)_{t}\left(\phi-\phi_{\Omega}\right) d x, & \text { if }\left.\nabla \phi \cdot v\right|_{\partial \Omega}=0, \phi_{\Omega}=\frac{1}{|\Omega|} \int_{\Omega} \phi d x .\end{cases}
\end{aligned}
$$

Here we have used the facts

$$
\begin{aligned}
\int_{\Omega} u \cdot \nabla(\nabla \cdot \varphi) d x & =-\int_{\Omega}(\nabla \cdot u)(\nabla \cdot \varphi) d x=\int_{\Omega}\left[\nabla \cdot \varphi_{t}+\nabla \cdot(u \cdot \nabla \varphi)\right](\nabla \cdot \varphi) d x \\
& =\frac{1}{2} \frac{d}{d t} \int_{\Omega}|\nabla \cdot \varphi|^{2} d x+\int_{\Omega} \nabla \cdot(u \cdot \nabla \varphi)(\nabla \cdot \varphi) d x ;
\end{aligned}
$$

and if $\left.\phi\right|_{\partial \Omega}=0$, then

$$
\begin{aligned}
-\int_{\Omega} u \cdot \nabla \phi d x & =\int_{\Omega}(\nabla \cdot u) \phi d x=\int_{\Omega}\left[-\nabla \cdot \varphi_{t}-\nabla \cdot(u \cdot \nabla \varphi)\right] \phi d x \\
& =-\int_{\Omega} \partial_{t}(\Delta \phi) \phi d x+\int_{\Omega}\left[O\left(|\nabla \varphi|^{2}\right)_{t} \phi+(u \cdot \nabla \varphi) \cdot \nabla \phi\right] d x \\
& =\frac{1}{2} \frac{d}{d t} \int_{\Omega}|\nabla \phi|^{2} d x+\int_{\Omega}\left[O\left(|\nabla \varphi|^{2}\right)_{t} \phi+(u \cdot \nabla \varphi) \cdot \nabla \phi\right] d x ;
\end{aligned}
$$

and if $\left.\nabla \phi \cdot v\right|_{\partial \Omega}=0$ (implying $\left.\nabla \phi_{t} \cdot v\right|_{\partial \Omega}=0$ ), then

$$
\begin{aligned}
-\int_{\Omega} u \cdot \nabla \phi d x & =-\int_{\Omega} u \cdot \nabla\left(\phi-\phi_{\Omega}\right) d x \\
& =\int_{\Omega}(\nabla \cdot u)\left(\phi-\phi_{\Omega}\right) d x=\int_{\Omega}\left[-\nabla \cdot \varphi_{t}-\nabla \cdot(u \cdot \nabla \varphi)\right]\left(\phi-\phi_{\Omega}\right) d x \\
& =-\int_{\Omega} \partial_{t}(\Delta \phi)\left(\phi-\phi_{\Omega}\right) d x+\int_{\Omega}\left[O\left(|\nabla \varphi|^{2}\right)_{t}\left(\phi-\phi_{\Omega}\right)+(u \cdot \nabla \varphi) \cdot \nabla \phi\right] d x \\
& =\frac{1}{2} \frac{d}{d t} \int_{\Omega}|\nabla \phi|^{2} d x+\int_{\Omega}\left[O\left(|\nabla \varphi|^{2}\right)_{t}\left(\phi-\phi_{\Omega}\right)+(u \cdot \nabla \varphi) \cdot \nabla \phi\right] d x .
\end{aligned}
$$

Then, noting (2.9), we easily use Hölder's inequality and Lemmas A.1-A.2 to bound the right-hand side of (3.3) by $\epsilon\left\|\left(\nabla u, \nabla \varphi, \nabla^{2} \varphi\right)\right\|^{2}$. 
Next, we construct the dissipation estimate for $\nabla u_{t}$.

Lemma 3.2. It holds that

$$
\frac{d}{d t}\left\|\left(u_{t}, \nabla \cdot \varphi_{t}, \nabla \varphi_{t}, \nabla \phi_{t}\right)\right\|^{2}+\left\|\nabla u_{t}\right\|^{2} \lesssim \epsilon\|\nabla u\|_{H^{1}}^{2}
$$

Proof. From

$$
\left\{\begin{array}{l}
\left.u\right|_{\partial \Omega}=\left.\varphi\right|_{\partial \Omega}=0, \\
\left.\phi\right|_{\partial \Omega}=0 \text { or }\left.\nabla \phi \cdot v\right|_{\partial \Omega}=0
\end{array}\right.
$$

we obtain

$$
\left\{\begin{array}{l}
\left.u_{t}\right|_{\partial \Omega}=\left.\varphi_{t}\right|_{\partial \Omega}=0, \\
\left.\phi_{t}\right|_{\partial \Omega}=0 \text { or }\left.\nabla \phi_{t t} \cdot v\right|_{\partial \Omega}=0 .
\end{array}\right.
$$

We integrate the resulting identity $u_{t} \cdot\left(\partial_{t} L_{1}-\partial_{t} R_{1}\right)-\Delta \varphi_{t} \cdot\left(\partial_{t} L_{2}-\partial_{t} R_{2}\right)=0$ over $\Omega$ to obtain

$$
\begin{aligned}
& \frac{1}{2} \frac{d}{d t} \int_{\Omega}\left(\left|u_{t}\right|^{2}+\left|\nabla \cdot \varphi_{t}\right|^{2}+\left|\nabla \varphi_{t}\right|^{2}+\left|\nabla \phi_{t}\right|^{2}\right) d x+\int_{\Omega}\left(\mu\left|\nabla u_{t}\right|^{2}+(\mu+\lambda)\left|\nabla \cdot u_{t}\right|^{2}\right) d x \\
& =\int_{\Omega}\left[\partial_{t} R_{1} \cdot u_{t}-\partial_{t} R_{2} \cdot \Delta \varphi_{t}-\nabla \cdot(u \cdot \nabla \varphi)_{t}(\nabla \cdot \varphi)_{t}-(u \cdot \nabla \varphi)_{t} \cdot \nabla \phi_{t}\right] d x \\
& \quad- \begin{cases}\int_{\Omega} O\left(|\nabla \varphi|^{2}\right)_{t t} \phi_{t} d x, & \text { if }\left.\phi_{t}\right|_{\partial \Omega}=0, \\
\int_{\Omega} O\left(|\nabla \varphi|^{2}\right)_{t t}\left(\phi_{t}-\phi_{t \Omega}\right) d x, & \text { if }\left.\nabla \phi_{t t} \cdot v\right|_{\partial \Omega}=0, \phi_{t \Omega}=\frac{1}{|\Omega|} \int_{\Omega} \phi_{t} d x .\end{cases}
\end{aligned}
$$

Here we have used the facts

$$
\begin{aligned}
\int_{\Omega} u_{t} \cdot \nabla(\nabla \cdot \varphi)_{t} d x & =-\int_{\Omega}(\nabla \cdot u)_{t}(\nabla \cdot \varphi)_{t} d x=\int_{\Omega}\left[\nabla \cdot \varphi_{t}+\nabla \cdot(u \cdot \nabla \varphi)\right]_{t}(\nabla \cdot \varphi)_{t} d x \\
& =\frac{1}{2} \frac{d}{d t} \int_{\Omega}\left|\nabla \cdot \varphi_{t}\right|^{2} d x+\int_{\Omega} \nabla \cdot(u \cdot \nabla \varphi)_{t}(\nabla \cdot \varphi)_{t} d x
\end{aligned}
$$

and if $\left.\phi_{t}\right|_{\partial \Omega}=0$, then

$$
\begin{aligned}
-\int_{\Omega} u_{t} \cdot \nabla \phi_{t} d x & =\int_{\Omega}(\nabla \cdot u)_{t} \phi_{t} d x=\int_{\Omega}\left[-\nabla \cdot \varphi_{t}-\nabla \cdot(u \cdot \nabla \varphi)\right]_{t} \phi_{t} d x \\
& =-\int_{\Omega} \partial_{t t}(\Delta \phi) \phi_{t} d x+\int_{\Omega} O\left(|\nabla \varphi|^{2}\right)_{t t} \phi_{t} d x+\int_{\Omega}(u \cdot \nabla \varphi)_{t} \cdot \nabla \phi_{t} d x \\
& =\frac{1}{2} \frac{d}{d t} \int_{\Omega}\left|\nabla \phi_{t}\right|^{2} d x+\int_{\Omega}\left[O\left(|\nabla \varphi|^{2}\right)_{t t} \phi_{t}+(u \cdot \nabla \varphi)_{t} \cdot \nabla \phi_{t}\right] d x ;
\end{aligned}
$$

and if $\left.\nabla \phi_{t t} \cdot v\right|_{\partial \Omega}=0$, then

$$
\begin{aligned}
-\int_{\Omega} u_{t} \cdot \nabla \phi_{t} d x & =-\int_{\Omega} u_{t} \cdot \nabla\left(\phi_{t}-\phi_{t \Omega}\right) d x \\
& =\int_{\Omega}(\nabla \cdot u)_{t}\left(\phi_{t}-\phi_{t \Omega}\right) d x=\int_{\Omega}\left[-\nabla \cdot \varphi_{t}-\nabla \cdot(u \cdot \nabla \varphi)\right]_{t}\left(\phi_{t}-\phi_{t \Omega}\right) d x \\
& =-\int_{\Omega} \partial_{t t}(\Delta \phi)\left(\phi_{t}-\phi_{t \Omega}\right) d x+\int_{\Omega} O\left(|\nabla \varphi|^{2}\right)_{t t}\left(\phi_{t}-\phi_{t \Omega}\right) d x+\int_{\Omega}(u \cdot \nabla \varphi)_{t} \cdot \nabla \phi_{t} d x
\end{aligned}
$$




$$
=\frac{1}{2} \frac{d}{d t} \int_{\Omega}\left|\nabla \phi_{t}\right|^{2} d x+\int_{\Omega}\left[O\left(|\nabla \varphi|^{2}\right)_{t t}\left(\phi_{t}-\phi_{t \Omega}\right)+(u \cdot \nabla \varphi)_{t} \cdot \nabla \phi_{t}\right] d x .
$$

Since $\sup _{0 \leqslant t \leqslant T}\|(u, \nabla \varphi, \nabla \phi)\|_{H^{2}}<\epsilon \ll 1$, by some straightforward calculations, we easily deduce from (2.8) that

$$
\left\{\begin{array}{l}
\left\|\left(\nabla \varphi_{t}, \nabla \phi_{t}, \nabla^{2} \phi_{t}\right)\right\| \lesssim\|\nabla u\|, \\
\left\|\nabla^{2} \varphi_{t}\right\| \lesssim\left\|\nabla^{2} u\right\|+\epsilon\|\nabla u\|_{H^{1}}, \\
\left\|\nabla \varphi_{t t}\right\| \lesssim\left\|\nabla u_{t}\right\|+\epsilon\|\nabla u\|_{H^{1}} .
\end{array}\right.
$$

Note that

$$
\partial_{t} R_{1} \approx u_{t} \cdot \nabla u+u \cdot \nabla u_{t}+\nabla \varphi_{t} \nabla^{2} u+\nabla \varphi \nabla^{2} u_{t}+\nabla \varphi_{t} \nabla^{2} \varphi+\nabla \varphi \nabla^{2} \varphi_{t}+\text { cubic terms. }
$$

Then, by (3.6)-(3.7), we can use Hölder's inequality and Lemmas A.1-A.2 to bound the right-hand side of (3.5) by $\epsilon\left(\|\nabla u\|_{H^{1}}^{2}+\left\|\nabla u_{t}\right\|^{2}\right)$.

The following estimate is very important since it gives the estimate independent of $\nabla^{2} u$ for the electric field $-\nabla \phi$.

Lemma 3.3. It holds that

$$
\|(\nabla \varphi, \nabla \phi)\|^{2} \lesssim\left\|\left(\nabla u_{t}, \nabla u\right)\right\|^{2}
$$

Proof. Multiplying $(2.8)_{1}$ by $-\varphi$ and integrating the resulting identity over $\Omega$ by parts, by Hölder's and Cauchy's inequalities and Lemmas A.1-A.2, we can obtain

$$
\int_{\Omega}\left(|\nabla \varphi|^{2}+|\nabla \cdot \varphi|^{2}+|\nabla \phi|^{2}\right) d x \lesssim\left\|\left(\nabla u_{t}, \nabla u\right)\right\|^{2} .
$$

Here we have estimated this term in light of different boundary conditions:

(1) If $\left.\phi\right|_{\partial \Omega}=0$, by Lemmas A.1-A.2, then

$$
\begin{aligned}
\int_{\Omega} \nabla \phi \cdot \varphi d x & =-\int_{\Omega} \phi(\nabla \cdot \varphi) d x \\
& =-\int_{\Omega} \phi \Delta \phi d x+\int_{\Omega} \phi O\left(|\nabla \varphi|^{2}\right) d x \\
& \gtrsim \int_{\Omega}|\nabla \phi|^{2} d x-\|\phi\|_{L^{\infty}}\|\nabla \varphi\|^{2} \\
& \gtrsim \int_{\Omega}|\nabla \phi|^{2} d x-\|\phi\|_{H^{2}}^{\frac{3}{4}}\|\phi\|^{\frac{1}{4}}\|\nabla \varphi\|^{2} \\
& \gtrsim \int_{\Omega}|\nabla \phi|^{2} d x-\|\nabla \phi\|_{H^{1}}\|\nabla \varphi\|^{2} \\
& \gtrsim \int_{\Omega}|\nabla \phi|^{2} d x-\epsilon\|\nabla \varphi\|^{2} .
\end{aligned}
$$

(2) If $\left.\nabla \phi \cdot v\right|_{\partial \Omega}=0$, by Lemmas A.1-A.2, then

$$
\begin{aligned}
\int_{\Omega} \nabla \phi \cdot \varphi d x & =\int_{\Omega} \nabla\left(\phi-\phi_{\Omega}\right) \cdot \varphi d x \\
& =-\int_{\Omega}\left(\phi-\phi_{\Omega}\right)(\nabla \cdot \varphi) d x
\end{aligned}
$$




$$
\begin{aligned}
& =-\int_{\Omega}\left(\phi-\phi_{\Omega}\right) \Delta \phi d x+\int_{\Omega}\left(\phi-\phi_{\Omega}\right) O\left(|\nabla \varphi|^{2}\right) d x \\
& \gtrsim \int_{\Omega}|\nabla \phi|^{2} d x-\left\|\phi-\phi_{\Omega}\right\|_{L^{\infty}}\|\nabla \varphi\|^{2} \\
& \gtrsim \int_{\Omega}|\nabla \phi|^{2} d x-\left\|\phi-\phi_{\Omega}\right\|_{H^{2}}^{\frac{3}{4}}\left\|\phi-\phi_{\Omega}\right\|^{\frac{1}{4}}\|\nabla \varphi\|^{2} \\
& \gtrsim \int_{\Omega}|\nabla \phi|^{2} d x-\|\nabla \phi\|_{H^{1}}\|\nabla \varphi\|^{2} \\
& \gtrsim \int_{\Omega}|\nabla \phi|^{2} d x-\epsilon\|\nabla \varphi\|^{2} .
\end{aligned}
$$

So, we deduce (3.8) from (3.9).

It is necessary to derive the following estimate so that the energy located under the time derivative contains $\nabla u$.

Lemma 3.4. It holds that

$$
\frac{d}{d t}\|(\nabla u, \nabla \cdot u)\|^{2}+\left\|u_{t}\right\|^{2} \lesssim\left\|\left(\nabla u_{t}, \nabla \varphi, \nabla \phi\right)\right\|^{2}+\epsilon\left\|\left(\nabla u, \nabla^{2} \varphi\right)\right\|^{2} .
$$

Proof. By integrating the resulting identity $u_{t} \times\left(L_{1}-R_{1}\right)=0$ over $\Omega$, we obtain

$$
\begin{aligned}
& \frac{1}{2} \frac{d}{d t} \int_{\Omega}\left(\mu|\nabla u|^{2}+(\mu+\lambda)|\nabla \cdot u|^{2}\right) d x+\int_{\Omega}\left|u_{t}\right|^{2} d x \\
& \quad=\int_{\Omega}\left(-u_{t} \cdot \Delta \varphi-u_{t} \cdot \nabla \nabla \cdot \varphi+u_{t} \cdot \nabla \phi+u_{t} \cdot R_{1}\right) d x \\
& \quad=\int_{\Omega}\left(\nabla u_{t}: \nabla \varphi+\nabla \cdot u_{t} \nabla \cdot \varphi+u_{t} \cdot \nabla \phi+u_{t} \cdot R_{1}\right) d x \\
& \quad \lesssim\left\|\nabla u_{t}\right\|\|\nabla \varphi\|+\left\|u_{t}\right\|\|\nabla \phi\|+\int_{\Omega} u_{t} \cdot R_{1} d x .
\end{aligned}
$$

By (2.9), Hölder's inequality and Lemmas A.1-A.2, we easily obtain

$$
\int_{\Omega} u_{t} \cdot R_{1} d x \lesssim \epsilon\left(\|\nabla u\|^{2}+\left\|\nabla u_{t}\right\|^{2}+\left\|\nabla^{2} \varphi\right\|^{2}\right) .
$$

Plugging (3.12) into (3.11), by Cauchy's inequality, we deduce (3.10).

So far, we have used the above four lemmas to establish the lower-order energy estimates for $(u, \varphi, \phi)$. To obtain the estimates of the higher-order derivatives of $(u, \varphi, \phi)$, we have to split the estimates into the interior estimates and the estimates near the boundary, cf. [36, 37]. We first establish the interior estimates.

Lemma 3.5. Let $\chi_{0} \in C_{0}^{\infty}(\Omega)$ be any fixed function. It holds that

$$
\begin{aligned}
& \frac{d}{d t}\left\|\chi_{0}(\nabla u, \nabla \nabla \cdot \varphi, \Delta \varphi, \Delta \phi)\right\|^{2}+\left\|\chi_{0} \nabla^{2} u\right\|^{2} \lesssim\|\nabla u\|^{2}+\epsilon\left(\left\|\left(\nabla^{2} \varphi, \nabla \phi\right)\right\|^{2}+\|\nabla u\|_{H^{1}}^{2}\right) ; \\
& \frac{d}{d t}\left\|\chi_{0}\left(\nabla^{2} u, \nabla^{2} \nabla \cdot \varphi, \nabla \Delta \varphi, \nabla \Delta \phi\right)\right\|^{2}+\left\|\chi_{0} \nabla^{3} u\right\|^{2} \lesssim\|\nabla u\|_{H^{1}}^{2}+\epsilon\left(\left\|\nabla^{2} \varphi\right\|_{H^{1}}^{2}+\|\nabla u\|_{H^{2}}^{2}+\left\|\nabla^{2} \phi\right\|^{2}\right) ; \\
& \left\|\chi_{0}\left(\nabla^{2} \varphi, \nabla^{2} \phi\right)\right\|^{2} \lesssim\left\|\left(\nabla \varphi, \nabla \phi, \nabla u_{t}, \chi_{0} \nabla^{2} u\right)\right\|^{2}+\epsilon\|\nabla u\|^{2} ; \\
& \left\|\chi_{0}\left(\nabla^{3} \varphi, \nabla^{3} \phi\right)\right\|^{2} \lesssim\left\|\left(\nabla \varphi, \nabla^{2} \varphi, \nabla^{2} \phi, \nabla u_{t}, \chi_{0} \nabla^{3} u\right)\right\|^{2}+\epsilon\|\nabla u\|_{H^{1}}^{2} .
\end{aligned}
$$


Proof. Integrating the identity $\nabla\left(L_{1}-R_{1}\right): \nabla u \chi_{0}^{2}+\nabla\left(L_{2}-R_{2}\right):\left(-\nabla \Delta \varphi \chi_{0}^{2}\right)=0$ over $\Omega$, we obtain

$$
\begin{aligned}
& \frac{1}{2} \frac{d}{d t} \int_{\Omega} \chi_{0}^{2}\left(|\nabla u|^{2}+|\nabla \nabla \cdot \varphi|^{2}+|\Delta \varphi|^{2}+|\Delta \phi|^{2}\right) d x+\int_{\Omega} \chi_{0}^{2}\left(\mu|\Delta u|^{2}+(\mu+\lambda)|\nabla \nabla \cdot u|^{2}\right) d x \\
& =-\mu \int_{\Omega} 2 \chi_{0} \Delta u \cdot \nabla u \cdot \nabla \chi_{0} d x-(\mu+\lambda) \int_{\Omega} 2 \chi_{0}\left[\nabla(\nabla \cdot u) \cdot \nabla u \cdot \nabla \chi_{0}-(\nabla \times u) \cdot \nabla(\nabla \cdot u) \times \nabla \chi_{0}\right] d x \\
& \quad-\int_{\Omega} \nabla(\nabla \cdot \varphi) \cdot \nabla(\nabla \cdot(u \cdot \nabla \varphi)) \chi_{0}^{2} d x+\int_{\Omega} 2 \chi_{0}\left[\nabla(\nabla \cdot \varphi) \cdot \nabla u \cdot \nabla \chi_{0}-(\nabla \times u) \cdot \nabla(\nabla \cdot \varphi) \times \nabla \chi_{0}\right] d x \\
& \quad-\int_{\Omega} 2 \chi_{0}\left[\nabla \phi \cdot \nabla u \cdot \nabla \chi_{0}-(\nabla \times u) \cdot \nabla \phi \times \nabla \chi_{0}\right] d x \\
& \quad-\int_{\Omega} 2 \chi_{0} \Delta \phi_{t} \nabla \phi \cdot \nabla \chi_{0}+\chi_{0}^{2} \nabla\left[O\left(|\nabla \varphi|^{2}\right)_{t}-\nabla \cdot(u \cdot \nabla \varphi)\right] \cdot \nabla \phi d x \\
& -\int_{\Omega} 2 \chi_{0} \nabla \varphi_{t} \cdot \Delta \varphi \cdot \nabla \chi_{0} d x+\int_{\Omega} \chi_{0}^{2}\left(\nabla R_{1}: \nabla u-\nabla R_{2}: \nabla \Delta \varphi\right) d x,
\end{aligned}
$$

where we have computed

$$
\begin{aligned}
& -\int_{\Omega} \nabla \nabla(\nabla \cdot u): \nabla u \chi_{0}^{2} d x=\int_{\Omega} \nabla(\nabla \cdot u) \cdot \Delta u \chi_{0}^{2} d x+\int_{\Omega} 2 \chi_{0} \nabla(\nabla \cdot u) \cdot \nabla u \cdot \nabla \chi_{0} d x \\
& =\int_{\Omega} \nabla(\nabla \cdot u) \cdot[\nabla(\nabla \cdot u)-\nabla \times(\nabla \times u)] \chi_{0}^{2} d x+\int_{\Omega} 2 \chi_{0} \nabla(\nabla \cdot u) \cdot \nabla u \cdot \nabla \chi_{0} d x \\
& =\int_{\Omega}\left|\nabla(\nabla \cdot u) \chi_{0}\right|^{2} d x-\int_{\Omega} \nabla \times(\nabla \times u) \cdot \nabla(\nabla \cdot u) \chi_{0}^{2} d x+\int_{\Omega} 2 \chi_{0} \nabla(\nabla \cdot u) \cdot \nabla u \cdot \nabla \chi_{0} d x \\
& =\int_{\Omega}\left|\nabla(\nabla \cdot u) \chi_{0}\right|^{2} d x+\int_{\Omega} 2 \chi_{0}\left[\nabla(\nabla \cdot u) \cdot \nabla u \cdot \nabla \chi_{0}-(\nabla \times u) \cdot \nabla(\nabla \cdot u) \times \nabla \chi_{0}\right] d x ; \\
& \int_{\Omega} \nabla^{2}(\nabla \cdot \varphi): \nabla u \chi_{0}^{2} d x=-\int_{\Omega} \nabla(\nabla \cdot \varphi) \cdot \nabla \cdot\left(\nabla u \chi_{0}^{2}\right) d x \\
& =-\int_{\Omega} 2 \chi_{0} \nabla(\nabla \cdot \varphi) \cdot \nabla u \cdot \nabla \chi_{0} d x-\int_{\Omega} \Delta u \cdot \nabla(\nabla \cdot \varphi) \chi_{0}^{2} d x \\
& =-\int_{\Omega} 2 \chi_{0} \nabla(\nabla \cdot \varphi) \cdot \nabla u \cdot \nabla \chi_{0} d x+\int_{\Omega} \nabla \times(\nabla \times u) \cdot \nabla(\nabla \cdot \varphi) \chi_{0}^{2} d x-\int_{\Omega} \nabla(\nabla \cdot u) \cdot \nabla(\nabla \cdot \varphi) \chi_{0}^{2} d x \\
& =-\int_{\Omega} 2 \chi_{0} \nabla(\nabla \cdot \varphi) \cdot \nabla u \cdot \nabla \chi_{0} d x+\int_{\Omega} 2 \chi_{0}(\nabla \times u) \cdot \nabla(\nabla \cdot \varphi) \times \nabla \chi_{0} d x-\int_{\Omega} \nabla(\nabla \cdot u) \cdot \nabla(\nabla \cdot \varphi) \chi_{0}^{2} d x \\
& =-\int_{\Omega} 2 \chi_{0} \nabla(\nabla \cdot \varphi) \cdot \nabla u \cdot \nabla \chi_{0} d x+\int_{\Omega} 2 \chi_{0}(\nabla \times u) \cdot \nabla(\nabla \cdot \varphi) \times \nabla \chi_{0} d x \\
& +\int_{\Omega} \nabla\left[(\nabla \cdot \varphi)_{t}+\nabla \cdot(u \cdot \nabla \varphi)\right] \cdot \nabla(\nabla \cdot \varphi) \chi_{0}^{2} d x \\
& =\frac{1}{2} \frac{d}{d t} \int_{\Omega} \chi_{0}^{2}|\nabla(\nabla \cdot \varphi)|^{2} d x+\int_{\Omega} \nabla(\nabla \cdot \varphi) \cdot \nabla[\nabla \cdot(u \cdot \nabla \varphi)] \chi_{0}^{2} d x \\
& -\int_{\Omega} 2 \chi_{0}\left[\nabla(\nabla \cdot \varphi) \cdot \nabla u \cdot \nabla \chi_{0}-(\nabla \times u) \cdot \nabla(\nabla \cdot \varphi) \times \nabla \chi_{0}\right] d x \\
& -\int_{\Omega} \nabla^{2} \phi: \nabla u \chi_{0}^{2} d x=\int_{\Omega} \nabla \phi \cdot \nabla \cdot\left(\nabla u \chi_{0}^{2}\right) d x
\end{aligned}
$$




$$
\begin{aligned}
= & \int_{\Omega} 2 \chi_{0} \nabla \phi \cdot \nabla u \cdot \nabla \chi_{0} d x+\int_{\Omega} \Delta u \cdot \nabla \phi \chi_{0}^{2} d x \\
= & \int_{\Omega} 2 \chi_{0} \nabla \phi \cdot \nabla u \cdot \nabla \chi_{0} d x+\int_{\Omega}[\nabla(\nabla \cdot u)-\nabla \times(\nabla \times u)] \cdot \nabla \phi \chi_{0}^{2} d x \\
= & \int_{\Omega} 2 \chi_{0} \nabla \phi \cdot \nabla u \cdot \nabla \chi_{0} d x-\int_{\Omega} \nabla \times(\nabla \times u) \cdot \nabla \phi \chi_{0}^{2} d x+\int_{\Omega} \nabla(\nabla \cdot u) \cdot \nabla \phi \chi_{0}^{2} d x \\
= & \int_{\Omega} 2 \chi_{0} \nabla \phi \cdot \nabla u \cdot \nabla \chi_{0} d x-\int_{\Omega} 2 \chi_{0}(\nabla \times u) \cdot \nabla \phi \times \nabla \chi_{0} d x+\int_{\Omega} \nabla(\nabla \cdot u) \cdot \nabla \phi \chi_{0}^{2} d x \\
= & \int_{\Omega} 2 \chi_{0} \nabla \phi \cdot \nabla u \cdot \nabla \chi_{0} d x-\int_{\Omega} 2 \chi_{0}(\nabla \times u) \cdot \nabla \phi \times \nabla \chi_{0} d x \\
& -\int_{\Omega} \nabla\left[(\nabla \cdot \varphi)_{t}+\nabla \cdot(u \cdot \nabla \varphi)\right] \cdot \nabla \phi \chi_{0}^{2} d x \\
= & \int_{\Omega} 2 \chi_{0} \nabla \phi \cdot \nabla u \cdot \nabla \chi_{0} d x-\int_{\Omega} 2 \chi_{0}(\nabla \times u) \cdot \nabla \phi \times \nabla \chi_{0} d x \\
& -\int_{\Omega} \nabla\left[\Delta \phi_{t}-O\left(|\nabla \varphi|^{2}\right)_{t}+\nabla \cdot(u \cdot \nabla \varphi)\right] \cdot \nabla \phi \chi_{0}^{2} d x \\
= & \frac{1}{2} \frac{d}{d t} \int_{\Omega} \chi_{0}^{2}|\Delta \phi|^{2} d x+\int_{\Omega} 2 \chi_{0}\left[\nabla \phi \cdot \nabla u \cdot \nabla \chi_{0}-(\nabla \times u) \cdot \nabla \phi \times \nabla \chi_{0}\right] d x \\
& +\int_{\Omega} 2 \chi_{0} \Delta \phi t \nabla \phi \cdot \nabla \chi_{0}+\nabla\left[O\left(|\nabla \varphi|^{2}\right)_{t}-\nabla \cdot(u \cdot \nabla \varphi)\right] \cdot \nabla \phi \chi_{0}^{2} d x .
\end{aligned}
$$

Then, by (3.6), Hölder's and Cauchy's inequalities and Lemmas A.1-A.2, we can bound the right-hand side of (3.17) by

$$
\epsilon\left(\left\|\nabla^{2} \varphi\right\|^{2}+\|\nabla u\|_{H^{1}}^{2}\right)+\|\nabla u\|\left\|\left(\nabla \phi, \nabla^{2} u, \nabla^{2} \varphi\right)\right\| .
$$

Note that

$$
\begin{aligned}
\int_{\Omega}\left|\nabla^{2} u\right|^{2} \chi_{0}^{2} d x=\int_{\Omega} \nabla^{2} u: \nabla^{2} u \chi_{0}^{2} d x \\
=-\int_{\Omega} \nabla u: \nabla \cdot\left(\nabla^{2} u \chi_{0}^{2}\right) d x \\
=-\int_{\Omega} \nabla u: \nabla \Delta u \chi_{0}^{2} d x-\int_{\Omega} 2 \chi_{0} \nabla u: \nabla^{2} u \cdot \nabla \chi_{0} d x \\
=\int_{\Omega}|\Delta u|^{2} \chi_{0}^{2} d x+\int_{\Omega} 2 \chi_{0} \nabla u \cdot \Delta u \cdot \nabla \chi_{0} d x-\int_{\Omega} 2 \chi_{0} \nabla u: \nabla^{2} u \cdot \nabla \chi_{0} d x \\
\lesssim \int_{\Omega}|\Delta u|^{2} \chi_{0}^{2} d x+\left\|\Delta u \chi_{0}\right\|\|\nabla u\|+\left\|\nabla^{2} u \chi_{0}\right\|\|\nabla u\| .
\end{aligned}
$$

By Cauchy's inequality, we have

$$
\int_{\Omega}\left|\nabla^{2} u\right|^{2} \chi_{0}^{2} d x \lesssim \int_{\Omega}|\Delta u|^{2} \chi_{0}^{2} d x+\|\nabla u\|^{2} .
$$

Thus we prove (3.13). Integrating the identity $\nabla^{2}\left(L_{1}-R_{1}\right): \nabla^{2} u \chi_{0}^{2}+\nabla^{2}\left(L_{2}-R_{2}\right):\left(-\nabla^{2} \Delta \varphi \chi_{0}^{2}\right)=0$ over $\Omega$, by the similar arguments as above, we easily obtain (3.14). 
Integrating the identity $\nabla\left(L_{1}-R_{1}\right): \nabla \varphi \chi_{0}^{2}=0$ over $\Omega$, by Hölder's and Cauchy's inequalities and Lemmas A.1-A.2, we obtain

$$
\begin{aligned}
\int_{\Omega} \chi_{0}^{2} & \left(|\Delta \varphi|^{2}+|\nabla \nabla \cdot \varphi|^{2}+|\Delta \phi|^{2}\right) d x \\
= & -\int_{\Omega} 2 \chi_{0} \Delta \varphi \cdot \nabla \varphi \cdot \nabla \chi_{0} d x-\int_{\Omega} 2 \chi_{0} \nabla(\nabla \cdot \varphi) \cdot\left[\nabla \varphi \cdot \nabla \chi_{0}-(\nabla \times \varphi) \times \nabla \chi_{0}\right] d x \\
& +\int_{\Omega} \Delta \phi O\left(|\nabla \varphi|^{2}\right) \chi_{0}^{2} d x+\int_{\Omega} 2 \chi_{0}\left[\nabla \phi \cdot \nabla \varphi \cdot \nabla \chi_{0}-(\nabla \cdot \varphi) \nabla \phi \cdot \nabla \chi_{0}-\nabla \phi \cdot(\nabla \times \varphi) \times \nabla \chi_{0}\right] d x \\
& +\int_{\Omega} \nabla\left[u_{t}-\mu \Delta u-(\mu+\lambda) \nabla(\nabla \cdot u)-R_{1}\right]: \nabla \varphi \chi_{0}^{2} d x \\
\lesssim & \|\nabla \varphi\|\left\|\Delta \varphi \chi_{0}\right\|+\|\nabla \varphi\|\left\|\nabla \nabla \cdot \varphi \chi_{0}\right\|+\left\|\Delta \phi \chi_{0}\right\|\|\nabla \varphi\|\|\nabla \varphi\|_{L^{\infty}}+\|\nabla \phi\|\|\nabla \varphi\| \\
& +\left\|\nabla u_{t}\right\|\|\nabla \varphi\|+\left\|\Delta u \chi_{0}\right\|\left\|\Delta \varphi \chi_{0}\right\|+\left\|\Delta u \chi_{0}\right\|\|\nabla \varphi\| \\
& +\left\|\nabla \nabla \cdot u \chi_{0}\right\|\left\|\Delta \varphi \chi_{0}\right\|+\left\|\nabla \nabla \cdot u \chi_{0}\right\|\|\nabla \varphi\| \\
& +\left\|\chi_{0} R_{1}\right\|\left\|\Delta \varphi \chi_{0}\right\|+\left\|\chi_{0} R_{1}\right\|\|\nabla \varphi\| \\
\lesssim & \left\|\left(\nabla \varphi, \nabla \phi, \nabla u_{t}, \Delta u \chi_{0}, \nabla \nabla \cdot u \chi_{0}\right)\right\|^{2}+\epsilon\left\|\left(\nabla u, \Delta \varphi \chi_{0}, \nabla \nabla \cdot \varphi \chi_{0}, \Delta \phi \chi_{0}\right)\right\|^{2},
\end{aligned}
$$

where we have computed

$$
\begin{aligned}
\int_{\Omega} & \nabla \Delta \varphi: \nabla \varphi \chi_{0}^{2} d x=-\int_{\Omega} \Delta \varphi \cdot \nabla \cdot\left(\nabla \varphi \chi_{0}^{2}\right) d x \\
& =-\int_{\Omega}|\Delta \varphi|^{2} \chi_{0}^{2} d x-\int_{\Omega} 2 \chi_{0} \Delta \varphi \cdot \nabla \varphi \cdot \nabla \chi_{0} d x \\
& \lesssim-\int_{\Omega}|\Delta \varphi|^{2} \chi_{0}^{2} d x+\left\|\Delta \varphi \chi_{0}\right\|\|\nabla \varphi\| ; \\
\int_{\Omega} & \nabla^{2}(\nabla \cdot \varphi): \nabla \varphi \chi_{0}^{2} d x=-\int_{\Omega} \nabla(\nabla \cdot \varphi) \cdot \nabla \cdot\left(\nabla \varphi \chi_{0}^{2}\right) d x \\
& =-\int_{\Omega} \nabla(\nabla \cdot \varphi) \cdot \Delta \varphi \chi_{0}^{2} d x-\int_{\Omega} 2 \chi_{0} \nabla(\nabla \cdot \varphi) \cdot \nabla \varphi \cdot \nabla \chi_{0} d x \\
& =-\int_{\Omega} \nabla(\nabla \cdot \varphi) \cdot[\nabla(\nabla \cdot \varphi)-\nabla \times(\nabla \times \varphi)] \chi_{0}^{2} d x-\int_{\Omega} 2 \chi_{0} \nabla(\nabla \cdot \varphi) \cdot \nabla \varphi \cdot \nabla \chi_{0} d x \\
& =-\int_{\Omega}|\nabla(\nabla \cdot \varphi)|^{2} \chi_{0}^{2} d x-\int_{\Omega} 2 \chi_{0} \nabla(\nabla \cdot \varphi) \cdot\left[\nabla \varphi \cdot \nabla \chi_{0}-(\nabla \times \varphi) \times \nabla \chi_{0}\right] d x \\
& \lesssim-\int_{\Omega}|\nabla(\nabla \cdot \varphi)|^{2} \chi_{0}^{2} d x+\left\|\nabla(\nabla \cdot \varphi) \chi_{0}\right\|\|\nabla \varphi\| ; \\
- & \int_{\Omega} \nabla^{2} \phi: \nabla \varphi \chi_{0}^{2} d x=\int_{\Omega} \nabla \phi \cdot \nabla \cdot\left(\nabla \varphi \chi_{0}^{2}\right) d x \\
& =\int_{\Omega} 2 \chi_{0} \nabla \phi \cdot \nabla \varphi \cdot \nabla \chi_{0} d x+\int_{\Omega} \nabla \phi \cdot \Delta \varphi \chi_{0}^{2} d x \\
& =\int_{\Omega} 2 \chi_{0} \nabla \phi \cdot \nabla \varphi \cdot \nabla \chi_{0} d x+\int_{\Omega} \nabla \phi \cdot[\nabla(\nabla \cdot \varphi)-\nabla \times(\nabla \times \varphi)] \chi_{0}^{2} d x \\
& =-\int_{\Omega} \Delta \phi(\nabla \cdot \varphi) \chi_{0}^{2} d x+\int_{\Omega} 2 \chi_{0}\left[\nabla \phi \cdot \nabla \varphi \cdot \nabla \chi_{0}-(\nabla \cdot \varphi) \nabla \phi \cdot \nabla \chi_{0}-\nabla \phi \cdot(\nabla \times \varphi) \times \nabla \chi_{0}\right] d x \\
& \\
& \\
&
\end{aligned}
$$




$$
\begin{aligned}
&=-\int_{\Omega}\left|\Delta \phi \chi_{0}\right|^{2} d x+\int_{\Omega} \Delta \phi O\left(|\nabla \varphi|^{2}\right) \chi_{0}^{2} d x \\
&+\int_{\Omega} 2 \chi_{0}\left[\nabla \phi \cdot \nabla \varphi \cdot \nabla \chi_{0}-(\nabla \cdot \varphi) \nabla \phi \cdot \nabla \chi_{0}-\nabla \phi \cdot(\nabla \times \varphi) \times \nabla \chi_{0}\right] d x \\
& \lesssim-\int_{\Omega}\left|\Delta \phi \chi_{0}\right|^{2} d x+\epsilon\left(\left\|\Delta \phi \chi_{0}\right\|^{2}+\|\nabla \varphi\|^{2}\right)+\|\nabla \phi\|\|\nabla \varphi\| ; \\
& \int_{\Omega} \nabla\left[-\mu \Delta u-(\mu+\lambda) \nabla(\nabla \cdot u)-R_{1}\right]: \nabla \varphi \chi_{0}^{2} d x \\
&=\int_{\Omega}\left[\mu \Delta u+(\mu+\lambda) \nabla(\nabla \cdot u)+R_{1}\right] \cdot \nabla \cdot\left(\nabla \varphi \chi_{0}^{2}\right) d x \\
&=\int_{\Omega}\left[\mu \Delta u+(\mu+\lambda) \nabla(\nabla \cdot u)+R_{1}\right] \cdot\left(\Delta \varphi \chi_{0}^{2}+2 \chi_{0} \nabla \varphi \cdot \nabla \chi_{0}\right) d x \\
& \lesssim\left\|\left[\mu \Delta u+(\mu+\lambda) \nabla(\nabla \cdot u)+R_{1}\right]\right\|\left(\left\|\Delta \varphi \chi_{0}\right\|+\|\nabla \varphi\|\right) .
\end{aligned}
$$

Hence, since $\epsilon \ll 1$, we deduce (3.15) from (3.18). Similarly, we can deduce (3.16) from $\int_{\Omega} \Delta\left(L_{1}-R_{1}\right) \cdot \Delta \varphi \chi_{0}^{2} d x=$ 0 , which is totally similar to (3.15).

Next, we shall construct the estimates of higher-order derivatives of $(u, \varphi, \phi)$ near the boundary, where we use a method introduced in [36,37]. The main idea is to straighten the boundary by introducing a suitable coordinate transformation on the restricted domain containing the boundary, thus we can integrate by parts to obtain the desired higher-order energy estimates since the tangential derivatives under new coordinates are always equal to zero on that flat boundary.

We shall choose a finite number of bounded open sets $\left\{\Theta_{j}\right\}_{j=1}^{N}$ in $\mathbb{R}^{3}$, such that $\partial \Omega \subset \cup_{j=1}^{N} \Theta_{j}$. The local coordinates $y=\left(y_{1}, y_{2}, y_{3}\right)$ in each open set $\Theta_{j}$ will satisfy the conditions as follows:

(1) The surface $\Theta_{j} \cap \partial \Omega$ is the image of a smooth vector function $z^{j}\left(y_{1}, y_{2}\right)=\left(z_{1}^{j}, z_{2}^{j}, z_{3}^{j}\right)\left(y_{1}, y_{2}\right)($ eg. take the local geodesic polar coordinate), satisfying

$$
\left|z_{y_{1}}^{j}\right|=1, z_{y_{1}}^{j} \cdot z_{y_{2}}^{j}=0 \text { and }\left|z_{y_{2}}^{j}\right| \geqslant \delta>0,
$$

where $\delta$ is some positive constant independent of $j, j=1,2, \ldots, N$.

(2) Any $x=\left(x_{1}, x_{2}, x_{3}\right) \in \Theta_{j}$ is expressed as

$$
x_{i}:=Y_{i}(y)=y_{3} \mathcal{N}_{i}^{j}\left(y_{1}, y_{2}\right)+z_{i}^{j}\left(y_{1}, y_{2}\right) \text { for } i=1,2,3,
$$

where $\mathcal{N}_{i}^{j}\left(y_{1}, y_{2}\right)$ denotes the unit outward normal vector at the boundary point with the coordinate $\left(y_{1}, y_{2}, 0\right)$.

We shall omit the superscript $j$ in what follows for simplicity without causing any misunderstanding. And we define the unit vectors

$$
e^{1}=z_{y_{1}} \quad \text { and } \quad e^{2}=\frac{z_{y_{2}}}{\left|z_{y_{2}}\right|},
$$

with $e^{1}=\left(e_{i}^{1}\right), e^{2}=\left(e_{i}^{2}\right), i=1,2$, 3. So, we have $\mathcal{N}=e^{1} \times e^{2}$.

By the Frenet-Serret's formula (cf. [5]), there exist smooth functions $\left(\alpha_{1}, \beta_{1}, \gamma_{1}, \alpha_{2}, \beta_{2}, \gamma_{2}\right)$ of $\left(y_{1}, y_{2}\right)$ satisfying

$$
\begin{gathered}
\frac{\partial}{\partial y_{1}}\left[\begin{array}{c}
e_{i}^{1} \\
e_{i}^{2} \\
\mathcal{N}_{i}
\end{array}\right]=\left[\begin{array}{ccc}
0 & -\gamma_{1} & -\alpha_{1} \\
\gamma_{1} & 0 & -\beta_{1} \\
\alpha_{1} & \beta_{1} & 0
\end{array}\right]\left[\begin{array}{l}
e_{i}^{1} \\
e_{i}^{2} \\
\mathcal{N}_{i}
\end{array}\right], \\
\frac{\partial}{\partial y_{2}}\left[\begin{array}{c}
e_{i}^{1} \\
e_{i}^{2} \\
\mathcal{N}_{i}
\end{array}\right]=\left[\begin{array}{ccc}
0 & -\gamma_{2} & -\alpha_{2} \\
\gamma_{2} & 0 & -\beta_{2} \\
\alpha_{2} & \beta_{2} & 0
\end{array}\right]\left[\begin{array}{c}
e_{i}^{1} \\
e_{i}^{2} \\
\mathcal{N}_{i}
\end{array}\right] .
\end{gathered}
$$


An easy computation shows that the Jacobian $J$ of the transform (3.19):

$$
J=Y_{y_{1}} \times Y_{y_{2}} \cdot \mathcal{N}=\left|z_{y_{2}}\right|+\left(\alpha_{1}\left|z_{y_{2}}\right|+\beta_{2}\right) y_{3}+\left(\alpha_{1} \beta_{2}-\beta_{1} \alpha_{2}\right) y_{3}^{2} .
$$

We observe that the transform (3.19) is regular through choosing $y_{3}$ so small that $J \geqslant \delta / 2$ from (3.20). Hence, the function $Y(y):=\left(Y_{1}, Y_{2}, Y_{3}\right)(y)$ is invertible. Moreover, the derivatives $\left(y_{1}, y_{2}, y_{3}\right)_{x_{i}}(x)$ make sense and can be expressed by

$$
\left\{\begin{array}{l}
\partial_{x_{i}} y_{1}=\frac{1}{J}\left(Y_{y_{2}} \times Y_{y_{3}}\right)_{i}=\frac{1}{J}\left(\mathcal{A} e_{i}^{1}+\mathcal{B} e_{i}^{2}\right)=: a_{1 i}, \\
\partial_{x_{i}} y_{2}=\frac{1}{J}\left(Y_{y_{3}} \times Y_{y_{1}}\right)_{i}=\frac{1}{J}\left(\mathcal{C} e_{i}^{1}+\mathcal{D} e_{i}^{2}\right)=: a_{2 i}, \\
\partial_{x_{i}} y_{3}=\frac{1}{J}\left(Y_{y_{1}} \times Y_{y_{2}}\right)_{i}=\mathcal{N}_{i}=: a_{3 i},
\end{array}\right.
$$

where $\mathcal{A}=\left|z_{y_{2}}\right|+\beta_{2} y_{3}, \mathcal{B}=-y_{3} \alpha_{2}, \mathcal{C}=-\beta_{1} y_{3}, \mathcal{D}=1+\alpha_{1} y_{3}$ and $J=\mathcal{A D}-\mathcal{B C} \geqslant \delta / 2$.

And we can deduce from (3.21) that

$$
\sum_{i=1}^{3} a_{1 i} a_{3 i}=\sum_{i=1}^{3} a_{2 i} a_{3 i}=0, \quad \sum_{i=1}^{3} a_{3 i}^{2}=|\mathcal{N}|^{2}=1
$$

and

$$
\partial_{x_{i}}=a_{1 i} \partial_{y_{1}}+a_{2 i} \partial_{y_{2}}+a_{3 i} \partial_{y_{3}}=\frac{1}{J}\left(\mathcal{A} e_{i}^{1}+\mathcal{B} e_{i}^{2}\right) \partial_{y_{1}}+\frac{1}{J}\left(\mathcal{C} e_{i}^{1}+\mathcal{D} e_{i}^{2}\right) \partial_{y_{2}}+\mathcal{N}_{i} \partial_{y_{3}} .
$$

Thus, in each $\Theta_{j},(2.7),(2.8)_{1}$ and $\nabla \cdot \varphi$ can be rewritten in the local coordinates $\left(y_{1}, y_{2}, y_{3}\right)$ as follows:

$$
\begin{aligned}
\mathcal{L}_{1}:= & \frac{D(\nabla \cdot \varphi)}{D t}+\frac{1}{J}\left[\left(\mathcal{A} e^{1}+\mathcal{B} e^{2}\right) \cdot u_{y_{1}}+\left(\mathcal{C} e^{1}+\mathcal{D} e^{2}\right) \cdot u_{y_{2}}+J \mathcal{N} \cdot u_{y_{3}}\right]=\mathcal{R}_{1}, \\
\mathcal{L}_{2}:= & u_{t}-\frac{\mu}{J^{2}}\left[\left(\mathcal{A}^{2}+\mathcal{B}^{2}\right) u_{y_{1} y_{1}}+2(\mathcal{A C}+\mathcal{B D}) u_{y_{1} y_{2}}+\left(\mathcal{C}^{2}+\mathcal{D}^{2}\right) u_{y_{2} y_{2}}+J^{2} u_{y_{3} y_{3}}\right] \\
& +(\text { first order terms of } u)+\frac{1}{J}\left(\mathcal{A} e^{1}+\mathcal{B} e^{2}\right)\left[(\mu+\lambda) \frac{D(\nabla \cdot \varphi)}{D t}+\nabla \cdot \varphi-\phi\right]_{y_{1}} \\
& +\frac{1}{J}\left(\mathcal{C} e^{1}+\mathcal{D} e^{2}\right)\left[(\mu+\lambda) \frac{D(\nabla \cdot \varphi)}{D t}+\nabla \cdot \varphi-\phi\right]_{y_{2}}+\mathcal{N}\left[(\mu+\lambda) \frac{D(\nabla \cdot \varphi)}{D t}+\nabla \cdot \varphi-\phi\right]_{y_{3}} \\
& +\frac{1}{J^{2}}\left[\left(\mathcal{A}^{2}+\mathcal{B}^{2}\right) \varphi_{y_{1} y_{1}}+2(\mathcal{A C}+\mathcal{B D}) \varphi_{y_{1} y_{2}}+\left(\mathcal{C}^{2}+\mathcal{D}^{2}\right) \varphi_{y_{2} y_{2}}+J^{2} \varphi_{y_{3} y_{3}}\right] \\
& +(\text { first order terms of } \varphi)=\mathcal{R}_{2}, \\
\nabla \cdot \varphi & \frac{1}{J}\left[\left(\mathcal{A} e^{1}+\mathcal{B} e^{2}\right) \cdot \varphi_{y_{1}}+\left(\mathcal{C} e^{1}+\mathcal{D} e^{2}\right) \cdot \varphi_{y_{2}}+J \mathcal{N} \cdot \varphi_{y_{3}}\right]=0,
\end{aligned}
$$

where

$$
\begin{gathered}
\mathcal{R}_{1}:=-(\nabla u)^{T}: \nabla \varphi, \\
\mathcal{R}_{2}:=-u \cdot \nabla u-\left(1-\frac{1}{\rho}\right)[\mu \Delta u+(\mu+\lambda) \nabla(\nabla \cdot u)]-\left(\frac{P^{\prime}(\rho)}{\rho}-1\right) \nabla\left[\nabla \cdot \varphi+O\left(|\nabla \varphi|^{2}\right)\right] \\
+O(|\nabla \varphi|) \nabla O(|\nabla \varphi|)+(\mu+\lambda) \nabla \mathcal{R}_{1} .
\end{gathered}
$$

We denote the tangential derivatives by $\partial=\left(\partial_{y_{1}}, \partial_{y_{2}}\right)$ and let $\chi_{j} \in C_{0}^{\infty}\left(\Theta_{j}\right)$ be any fixed function. Then

$$
\left\{\begin{array}{l}
\left.\chi_{j} \partial^{k} u\right|_{\partial \Omega_{j}^{-1}}=\left.\chi_{j} \partial^{k} \varphi\right|_{\partial \Omega_{j}^{-1}}=0, \\
\left.\chi_{j} \partial^{k} \phi\right|_{\partial \Omega_{j}^{-1}}=0 \text { or }\left.\chi_{j} \partial^{k} \nabla \phi \cdot v\right|_{\partial \Omega_{j}^{-1}}=0,
\end{array}\right.
$$

where $0 \leqslant k \leqslant 2$ and $\Omega_{j}^{-1}:=\left\{y \mid y=Y^{-1}(x), x \in \Omega_{j}=\Theta_{j} \cap \Omega\right\}$.

Next, we will use the following four lemmas (Lemmas 3.6-3.9) to give the energy estimates near the boundary. Then we can obtain the desired higher-order estimates for $(u, \varphi)$ (Lemma 3.10). 
Lemma 3.6. Let $\chi_{j} \in C_{0}^{\infty}\left(\Theta_{j}\right)$ be any fixed function. It holds that

$$
\begin{aligned}
& \frac{d}{d t}\left\|\chi_{j}(\partial u, \partial \nabla \cdot \varphi, \partial \nabla \varphi, \partial \nabla \phi)\right\|^{2}+\left\|\chi_{j} \partial \nabla u\right\|^{2} \lesssim\|\nabla u\|^{2}+\epsilon\left(\left\|\left(\nabla^{2} \varphi, \nabla \phi\right)\right\|^{2}+\|\nabla u\|_{H^{1}}^{2}\right) ; \\
& \frac{d}{d t}\left\|\chi_{j}\left(\partial^{2} u, \partial^{2} \nabla \cdot \varphi, \partial^{2} \nabla \varphi, \partial^{2} \nabla \phi\right)\right\|^{2}+\left\|\chi_{j} \partial^{2} \nabla u\right\|^{2} \lesssim\|\nabla u\|_{H^{1}}^{2}+\epsilon\left(\left\|\nabla^{2} \varphi\right\|_{H^{1}}^{2}+\|\nabla u\|_{H^{2}}^{2}+\left\|\nabla^{2} \phi\right\|^{2}\right) ; \\
& \left\|\chi_{j}(\partial \nabla \varphi, \partial \nabla \phi)\right\|^{2} \lesssim\left\|\left(\nabla \varphi, \nabla \phi, \nabla u_{t}, \chi_{j} \partial \nabla u\right)\right\|^{2}+\epsilon\|\nabla u\|^{2} ; \\
& \left\|\chi_{j}\left(\partial^{2} \nabla \varphi, \partial^{2} \nabla \phi\right)\right\|^{2} \lesssim\left\|\left(\nabla \varphi, \nabla^{2} \varphi, \nabla^{2} \phi, \nabla u_{t}, \chi_{j} \partial^{2} \nabla u\right)\right\|^{2}+\epsilon\|\nabla u\|_{H^{1}}^{2}
\end{aligned}
$$

Proof. It is similar to the proof of Lemma 3.5, so we omit it.

Lemma 3.7. Let $\chi_{j} \in C_{0}^{\infty}\left(\Theta_{j}\right)$ be any fixed function. It holds that

$$
\frac{d}{d t}\left\|\chi_{j}(\partial \nabla u, \partial \nabla \cdot u)\right\|^{2}+\left\|\chi_{j} \partial u_{t}\right\|^{2} \lesssim\left\|\left(\nabla^{2} \phi, \nabla^{3} \varphi, \nabla^{2} u\right)\right\|^{2}+\epsilon\left(\left\|\nabla^{3} u\right\|^{2}+\left\|\nabla^{2} \varphi\right\|^{2}\right) .
$$

Proof. Integrating the identity $\partial\left(L_{1}-R_{1}\right): \partial u_{t} \chi_{j}^{2}=0$ over $\Omega_{j}^{-1}$, by Hölder's and Cauchy's inequalities and Lemmas A.1-A.2, we have

$$
\begin{aligned}
& \frac{1}{2} \frac{d}{d t} \int_{\Omega_{j}^{-1}} \chi_{j}^{2}\left[\mu|\partial \nabla u|^{2}+(\mu+\lambda)|\partial \nabla \cdot u|^{2}\right] d y+\int_{\Omega_{j}^{-1}}\left|\chi_{j} \partial u_{t}\right|^{2} d y \\
& =-\int_{\Omega_{j}^{-1}} \chi_{j}^{2} \partial u_{t}: \partial(\nabla \nabla \cdot \varphi+\Delta \varphi-\nabla \phi) d y+\int_{\Omega_{j}^{-1}} \chi_{j}^{2} \partial u_{t}: \partial R_{1} d y \\
& \quad-\mu \int_{\Omega_{j}^{-1}} 2 \chi_{j} \partial \nabla u \cdot \partial u_{t} \cdot \nabla \chi_{j} d y-(\mu+\lambda) \int_{\Omega_{j}^{-1}} 2 \chi_{j} \partial \nabla \cdot u \cdot \partial u_{t} \cdot \nabla \chi_{j} d y \\
& \lesssim \epsilon\left(\left\|\chi_{j} \partial u_{t}\right\|^{2}+\left\|\nabla^{3} u\right\|^{2}+\left\|\nabla^{2} \varphi\right\|^{2}\right)+\left\|\nabla^{2} \phi\right\|^{2}+\left\|\nabla^{3} \varphi\right\|^{2}+\left\|\nabla^{2} u\right\|^{2} .
\end{aligned}
$$

We immediately deduce (3.27) from the above.

Lemma 3.8. Let $\chi_{j} \in C_{0}^{\infty}\left(\Theta_{j}\right)$ be any fixed function. It holds that

$$
\begin{aligned}
& \frac{d}{d t}\left\|\chi_{j} \partial_{y_{3}}(\nabla \cdot \varphi)\right\|^{2}+\left\|\chi_{j} \partial_{y_{3}}\left(\frac{D(\nabla \cdot \varphi)}{D t}\right)\right\|^{2}+\left\|\chi_{j} \partial_{y_{3}}(\nabla \cdot \varphi)\right\|^{2} \\
& \quad \lesssim\left\|\left(\nabla u, \nabla \varphi, \nabla \phi, u_{t}\right)\right\|^{2}+\left\|\chi_{j}(\partial \nabla u, \partial \nabla \varphi)\right\|^{2}+\epsilon\left\|\left(\nabla u, \nabla^{2} \varphi\right)\right\|_{H^{1}}^{2}
\end{aligned}
$$

and

$$
\begin{aligned}
& \frac{d}{d t}\left\|\chi_{j} \partial^{\kappa} \partial_{y_{3}}^{l+1}(\nabla \cdot \varphi)\right\|^{2}+\left\|\chi_{j} \partial^{\kappa} \partial_{y_{3}}^{l+1}\left(\frac{D(\nabla \cdot \varphi)}{D t}\right)\right\|^{2}+\left\|\chi_{j} \partial^{\kappa} \partial_{y_{3}}^{l+1}(\nabla \cdot \varphi)\right\|^{2} \\
& \quad \lesssim\left\|\left(\nabla u, \nabla \varphi, \nabla \phi, u_{t}\right)\right\|_{H^{1}}^{2}+\left\|\chi_{j}\left(\partial^{\kappa+1} \partial_{y_{3}}^{l} \nabla u, \partial^{\kappa+1} \partial_{y_{3}}^{l} \nabla \varphi\right)\right\|^{2}+\epsilon\|(\nabla u, \nabla \varphi)\|_{H^{2}}^{2},
\end{aligned}
$$

where $\kappa+\iota=1$.

Proof. Applying $\partial_{y_{3}}$ to $\mathcal{L}_{1}-\mathcal{R}_{1}=0$ and $\partial_{y_{3}}$ to (3.22), respectively, by $\mathcal{N} \cdot\left(\mathcal{L}_{2}-\mathcal{R}_{2}\right)=0$, we have

$$
\begin{aligned}
& \partial_{y_{3}}\left(\frac{D(\nabla \cdot \varphi)}{D t}\right)+\frac{1}{J}\left[\left(\mathcal{A} e^{1}+\mathcal{B} e^{2}\right) \cdot u_{y_{1} y_{3}}+\left(\mathcal{C} e^{1}+\mathcal{D} e^{2}\right) \cdot u_{y_{2} y_{3}}+J \mathcal{N} \cdot u_{y_{3} y_{3}}\right]+O(\nabla u)=\left(\mathcal{R}_{1}\right)_{y_{3}} ; \\
& \mathcal{N} \cdot u_{t}-\frac{\mu}{J^{2}}\left[\left(\mathcal{A}^{2}+\mathcal{B}^{2}\right) \mathcal{N} \cdot u_{y_{1} y_{1}}+2(\mathcal{A C}+\mathcal{B D}) \mathcal{N} \cdot u_{y_{1} y_{2}}+\left(\mathcal{C}^{2}+\mathcal{D}^{2}\right) \mathcal{N} \cdot u_{y_{2} y_{2}}+J^{2} \mathcal{N} \cdot u_{y_{3} y_{3}}\right] \\
& \quad+\frac{1}{J^{2}}\left[\left(\mathcal{A}^{2}+\mathcal{B}^{2}\right) \mathcal{N} \cdot \varphi_{y_{1} y_{1}}+2(\mathcal{A C}+\mathcal{B D}) \mathcal{N} \cdot \varphi_{y_{1} y_{2}}+\left(\mathcal{C}^{2}+\mathcal{D}^{2}\right) \mathcal{N} \cdot \varphi_{y_{2} y_{2}}+J^{2} \mathcal{N} \cdot \varphi_{y_{3} y_{3}}\right] \\
& \quad+\left[(\mu+\lambda) \frac{D(\nabla \cdot \varphi)}{D t}+\nabla \cdot \varphi-\phi\right]_{y_{3}}+O(\nabla u)+O(\nabla \varphi)=\mathcal{N} \cdot \mathcal{R}_{2} ; \\
& \frac{1}{J}\left[\left(\mathcal{A} e^{1}+\mathcal{B} e^{2}\right) \cdot \varphi_{y_{1} y_{3}}+\left(\mathcal{C} e^{1}+\mathcal{D} e^{2}\right) \varphi_{y_{2} y_{3}}+J \mathcal{N} \cdot \varphi_{y_{3} y_{3}}\right]-(\nabla \cdot \varphi)_{y_{3}}+O(\nabla \varphi)=0 .
\end{aligned}
$$

In order to eliminate the terms $\mathcal{N} \cdot u_{y_{3} y_{3}}$ and $\mathcal{N} \cdot \varphi_{y_{3} y_{3}}$ in (3.31), we compute $\mu \times(3.30)+(3.31)-(3.32)$ to obtain

$$
(2 \mu+\lambda) \partial_{y_{3}}\left(\frac{D(\nabla \cdot \varphi)}{D t}\right)+2 \partial_{y_{3}}(\nabla \cdot \varphi)
$$




$$
\begin{aligned}
= & \frac{\mu}{J^{2}}\left[\left(\mathcal{A}^{2}+\mathcal{B}^{2}\right) \mathcal{N} \cdot u_{y_{1} y_{1}}+2(\mathcal{A C}+\mathcal{B D}) \mathcal{N} \cdot u_{y_{1} y_{2}}+\left(\mathcal{C}^{2}+\mathcal{D}^{2}\right) \mathcal{N} \cdot u_{y_{2} y_{2}}\right] \\
& -\mathcal{N} \cdot u_{t}+\phi_{y_{3}}-\mu_{J}^{\mu}\left[\left(\mathcal{A} e^{1}+\mathcal{B} e^{2}\right) \cdot u_{y_{1} y_{3}}+\left(\mathcal{C} e^{1}+\mathcal{D} e^{2}\right) \cdot u_{y_{2} y_{3}}\right] \\
& +O(\nabla u)-\frac{1}{J^{2}}\left[\left(\mathcal{A}^{2}+\mathcal{B}^{2}\right) \mathcal{N} \cdot \varphi_{y_{1} y_{1}}+2(\mathcal{A C}+\mathcal{B D}) \mathcal{N} \cdot \varphi_{y_{1} y_{2}}+\left(\mathcal{C}^{2}+\mathcal{D}^{2}\right) \mathcal{N} \cdot \varphi_{y_{2} y_{2}}\right] \\
& +\frac{1}{J}\left[\left(\mathcal{A} e^{1}+\mathcal{B} e^{2}\right) \cdot \varphi_{y_{1} y_{3}}+\left(\mathcal{C} e^{1}+\mathcal{D} e^{2}\right) \cdot \varphi_{y_{2} y_{3}}\right] \\
& +O(\nabla \varphi)+\mu\left(\mathcal{R}_{1}\right)_{y_{3}}+\mathcal{N} \mathcal{R}_{2}:=\mathcal{R} .
\end{aligned}
$$

Multiplying (3.33) by $\chi_{j}^{2} \partial_{y_{3}}\left(\frac{D(\nabla \cdot \varphi)}{D t}+(\nabla \cdot \varphi)\right)$ and then integrating it over $\Omega_{j}^{-1}$, we obtain

$$
\begin{aligned}
& \frac{2+2 \mu+\lambda}{2} \frac{d}{d t}\left\|\chi_{j} \partial_{y_{3}}(\nabla \cdot \varphi)\right\|^{2}+(2 \mu+\lambda)\left\|\chi_{j} \partial_{y_{3}}\left(\frac{D(\nabla \cdot \varphi)}{D t}\right)\right\|^{2}+2\left\|\chi_{j} \partial_{y_{3}}(\nabla \cdot \varphi)\right\|^{2} \\
& \quad=-(2+2 \mu+\lambda) \int_{\Omega_{j}^{-1}}[u \cdot \nabla(\nabla \cdot \varphi)]_{y_{3}}(\nabla \cdot \varphi)_{y_{3}} \chi_{j}^{2} d y+\int_{\Omega_{j}^{-1}} \chi_{j}^{2} \partial_{y_{3}}\left[\frac{D(\nabla \cdot \varphi)}{D t}+(\nabla \cdot \varphi)\right] \mathcal{R} d y \\
& \quad:=I_{1}+I_{2} .
\end{aligned}
$$

We easily estimate the right-hand side of (3.34) as follows:

$$
\begin{aligned}
I_{1} & \lesssim \int_{\Omega_{j}^{-1}} \chi_{j}^{2}\left|u_{y_{3}} \cdot \nabla(\nabla \cdot \varphi)(\nabla \cdot \varphi)_{y_{3}}\right| d y+\int_{\Omega_{j}^{-1}}\left|(\nabla \cdot \varphi)_{y_{3}}^{2} \nabla \cdot\left(u \chi_{j}^{2}\right)\right| d y \\
& \lesssim\|\nabla u\|_{H^{1}}\|\nabla(\nabla \cdot \varphi)\|_{H^{1}}^{2} \lesssim \epsilon\|\nabla(\nabla \cdot \varphi)\|_{H^{1}}^{2}
\end{aligned}
$$

and

$$
\begin{aligned}
I_{2} \leqslant & \frac{2 \mu+\lambda}{2}\left\|\chi_{j} \partial_{y_{3}}\left(\frac{D(\nabla \cdot \varphi)}{D t}\right)\right\|^{2}+\left\|\chi_{j} \partial_{y_{3}}(\nabla \cdot \varphi)\right\|^{2}+C\left\|\chi_{j} \mathcal{R}\right\|^{2} \\
\leqslant & \frac{2 \mu+\lambda}{2}\left\|\chi_{j} \partial_{y_{3}}\left(\frac{D(\nabla \cdot \varphi)}{D t}\right)\right\|^{2}+\left\|\chi_{j} \partial_{y_{3}}(\nabla \cdot \varphi)\right\|^{2} \\
& +C\left\|\left(\nabla u, \nabla \varphi, \nabla \phi, u_{t}\right)\right\|^{2}+\epsilon\left\|\left(\nabla u, \nabla \varphi, \nabla^{2} \varphi\right)\right\|_{H^{1}}^{2}+C\left\|\chi_{j}(\partial \nabla u, \partial \nabla \varphi)\right\|^{2} .
\end{aligned}
$$

Substituting (3.35)-(3.36) into (3.34), we obtain (3.28).

Finally, applying $\partial^{k} \partial_{y_{3}}^{l}(k+\iota=1)$ to (3.33), multiplying the identity by $\partial^{k} \partial_{y_{3}}^{l+1}\left(\frac{D(\nabla \cdot \varphi)}{D t}+\nabla \cdot \varphi\right) \chi_{j}^{2}$, integrating over $\Omega_{j}^{-1}$ and as in the proof of (3.28), we can obtain (3.29) similarly.

Lemma 3.9. Let $\chi_{j} \in C_{0}^{\infty}\left(\Theta_{j}\right)$ be any fixed function. It holds that

$$
\begin{aligned}
\left\|\nabla^{2}\left(u-\frac{\varphi}{\mu}\right)\right\|^{2} \lesssim\left\|\left(u_{t}, \nabla \phi, \nabla u, \chi_{0} \nabla^{2} u, \chi_{j} \partial \nabla u, \chi_{j} \partial_{y_{3}}\left(\frac{D(\nabla \cdot \varphi)}{D t}\right)\right)\right\|^{2}+\|\nabla \cdot \varphi\|_{H^{1}}^{2}+\epsilon\left(\left\|\nabla^{2} u\right\|^{2}+\|\nabla \varphi\|_{H^{1}}^{2}\right) ; \\
\left\|\nabla^{3}\left(u-\frac{\varphi}{\mu}\right)\right\|^{2} \lesssim\left\|\left(u_{t}, \nabla \phi, \nabla u\right)\right\|_{H^{1}}^{2}+\left\|\left(\chi_{0} \nabla^{3} u, \chi_{j} \partial \nabla^{2} u, \chi_{j} \partial_{y_{3}} \nabla\left(\frac{D(\nabla \cdot \varphi)}{D t}\right)\right)\right\|^{2} \\
+\|\nabla \cdot \varphi\|_{H^{2}}^{2}+\epsilon\left(\|\nabla u\|_{H^{2}}^{2}+\left\|\nabla^{2} \varphi\right\|_{H^{1}}^{2}\right) ; \\
\left\|\chi_{j} \partial \nabla^{2}\left(u-\frac{\varphi}{\mu}\right)\right\|^{2} \lesssim\left\|\left(u_{t}, \nabla u, \nabla \varphi\right)\right\|_{H^{1}}^{2}+\|\nabla \phi\|^{2}+\left\|\chi_{j}\left(\partial^{2} \nabla u, \partial \partial_{y_{3}}\left(\frac{D(\nabla \cdot \varphi)}{D t}\right), \partial^{2}(\nabla \cdot \varphi), \partial \partial_{y_{3}}(\nabla \cdot \varphi)\right)\right\|^{2} \\
+\epsilon\left(\|\nabla u\|_{H^{2}}^{2}+\left\|\nabla^{2} \varphi\right\|_{H^{1}}^{2}\right) .
\end{aligned}
$$

Proof. By (2.7) and (2.8) $)_{1}$, we have

$$
\left\{\begin{array}{l}
\nabla \cdot\left(u-\frac{\varphi}{\mu}\right)=-\frac{D(\nabla \cdot \varphi)}{D t}-(\nabla u)^{T}: \nabla \varphi-\frac{1}{\mu} \nabla \cdot \varphi, \\
-\mu \Delta\left(u-\frac{\varphi}{\mu}\right)=-u_{t}+(\mu+\lambda) \nabla(\nabla \cdot u)-\nabla(\nabla \cdot \varphi)+\nabla \phi+R_{1}, \\
\left.\left(u-\frac{\varphi}{\mu}\right)\right|_{\partial \Omega}=0 .
\end{array}\right.
$$

Applying Lemma A.4 to (3.40), we obtain

$$
\left\|\nabla^{2}\left(u-\frac{\varphi}{\mu}\right)\right\|^{2}
$$




$$
\begin{aligned}
& \lesssim\left\|\left(u_{t}, \nabla \phi, R_{1}\right)\right\|^{2}+\left\|\frac{D(\nabla \cdot \varphi)}{D t}\right\|_{H^{1}}^{2}+\|\nabla \cdot \varphi\|_{H^{1}}^{2}+\left\|(\nabla u)^{T}: \nabla \varphi\right\|_{H^{1}}^{2} \\
& \lesssim\left\|\left(u_{t}, \nabla \phi\right)\right\|^{2}+\left\|\frac{D(\nabla \cdot \varphi)}{D t}\right\|_{H^{1}}^{2}+\|\nabla \cdot \varphi\|_{H^{1}}^{2}+\|u\|_{H^{1}}^{2}\left\|\nabla^{2} u\right\|^{2}+\|\nabla \varphi\|_{H^{1}}^{2}\|\nabla \varphi\|_{H^{2}}^{2}+\|\nabla \varphi\|_{H^{2}}^{2}\left\|\nabla^{2} u\right\|^{2} \\
& \lesssim\left\|\left(u_{t}, \nabla \phi\right)\right\|^{2}+\left\|\frac{D(\nabla \cdot \varphi)}{D t}\right\|_{H^{1}}^{2}+\|\nabla \cdot \varphi\|_{H^{1}}^{2}+\epsilon\left(\left\|\nabla^{2} u\right\|^{2}+\|\nabla \varphi\|_{H^{1}}^{2}\right),
\end{aligned}
$$

where we have estimated

$$
\|\nabla(\nabla \cdot u)\| \leqslant\left\|\nabla\left(\frac{D(\nabla \cdot \varphi)}{D t}\right)\right\|+\left\|\nabla\left[(\nabla u)^{T}: \nabla \varphi\right]\right\| .
$$

From $(3.40)_{1}$, we deduce

$$
\left\|\frac{D(\nabla \cdot \varphi)}{D t}\right\| \leqslant\|\nabla \cdot u\|+\left\|(\nabla u)^{T}: \nabla \varphi\right\| \lesssim\|\nabla u\|
$$

and

$$
\begin{aligned}
\left\|\nabla\left(\frac{D(\nabla \cdot \varphi)}{D t}\right)\right\| & \lesssim\left\|\chi_{0} \nabla\left(\frac{D(\nabla \cdot \varphi)}{D t}\right)\right\|+\left\|\chi_{j} \partial\left(\frac{D(\nabla \cdot \varphi)}{D t}\right)\right\|+\left\|\chi_{j} \partial_{y_{3}}\left(\frac{D(\nabla \cdot \varphi)}{D t}\right)\right\| \\
& \lesssim\left\|\chi_{0} \nabla^{2} u\right\|+\left\|\chi_{j} \partial \nabla u\right\|+\left\|\chi_{j} \partial_{y_{3}}\left(\frac{D(\nabla \cdot \varphi)}{D t}\right)\right\|+\left\|\nabla\left[(\nabla u)^{T}: \nabla \varphi\right]\right\| \\
& \lesssim\left\|\chi_{0} \nabla^{2} u\right\|+\left\|\chi_{j} \partial \nabla u\right\|+\left\|\chi_{j} \partial_{y_{3}}\left(\frac{D(\nabla \cdot \varphi)}{D t}\right)\right\|+\epsilon\|\nabla u\|_{H^{1}} .
\end{aligned}
$$

Thus we can deduce (3.37) from (3.41)-(3.43). Similarly, we can obtain

$$
\begin{aligned}
\left\|\nabla^{3}\left(u-\frac{\varphi}{\mu}\right)\right\|^{2} \lesssim & \left\|\left(u_{t}, \nabla \phi\right)\right\|_{H^{1}}^{2}+\left\|\frac{D(\nabla \cdot \varphi)}{D t}\right\|_{H^{2}}^{2}+\|\nabla \cdot \varphi\|_{H^{2}}^{2}+\epsilon\left(\|\nabla u\|_{H^{2}}^{2}+\left\|\nabla^{2} \varphi\right\|_{H^{1}}^{2}\right) \\
\lesssim & \left\|\left(u_{t}, \nabla \phi, \nabla u\right)\right\|_{H^{1}}^{2}+\left\|\chi_{0} \nabla^{3} u\right\|^{2}+\left\|\chi_{j} \partial \nabla^{2} u\right\|^{2}+\left\|\chi_{j} \partial_{y_{3}} \nabla\left(\frac{D(\nabla \cdot \varphi)}{D t}\right)\right\|^{2} \\
& +\|\nabla \cdot \varphi\|_{H^{2}}^{2}+\epsilon\left(\|\nabla u\|_{H^{2}}^{2}+\left\|\nabla^{2} \varphi\right\|_{H^{1}}^{2}\right) .
\end{aligned}
$$

To estimate the term $\left\|\chi_{j} \partial \nabla^{2} u\right\|^{2}$ on the right-hand side of (3.44), we need to apply $\chi_{j} \partial$ to (2.7) and (2.8) ${ }_{1}$ to obtain

$$
\left\{\begin{aligned}
\nabla \cdot\left(\chi_{j} \partial\left(u-\frac{\varphi}{\mu}\right)\right)= & -\chi_{j} \partial\left(\frac{D(\nabla \cdot \varphi)}{D t}\right)+\nabla \chi_{j} \cdot \partial u-\chi_{j} \partial\left[(\nabla u)^{T}: \nabla \varphi\right]-\frac{1}{\mu} \nabla \cdot\left(\chi_{j} \partial \varphi\right), \\
-\mu \Delta\left[\chi_{j} \partial\left(u-\frac{\varphi}{\mu}\right)\right]= & -2 \mu \nabla \chi_{j} \cdot \nabla\left[\partial\left(u-\frac{\varphi}{\mu}\right)\right]-\mu \Delta \chi_{j} \partial\left(u-\frac{\varphi}{\mu}\right) \\
& +\chi_{j} \partial\left[-u_{t}+(\mu+\lambda) \nabla(\nabla \cdot u)-\nabla(\nabla \cdot \varphi)+\nabla \phi+R_{1}\right], \\
\left.\chi_{j} \partial\left(u-\frac{\varphi}{\mu}\right)\right|_{\partial \Omega_{j}^{-1}=} & 0 .
\end{aligned}\right.
$$

Then applying Lemma A.4 to (3.45), we obtain

$$
\begin{aligned}
& \left\|\chi_{j} \partial \nabla^{2}\left(u-\frac{\varphi}{\mu}\right)\right\|^{2} \\
& \lesssim\left\|\left(\chi_{j} \partial u_{t}, \chi_{j} \partial \nabla \phi, \chi_{j} \partial R_{1}\right)\right\|^{2}+\left\|\chi_{j} \partial\left(\frac{D(\nabla \cdot \varphi)}{D t}\right)\right\|_{H^{1}}^{2}+\left\|\nabla \chi_{j} \cdot \partial u\right\|_{H^{1}}^{2}+\left\|\chi_{j} \partial\left[(\nabla u)^{T}: \nabla \varphi\right]\right\|_{H^{1}}^{2} \\
& \quad+\left\|\partial \nabla\left(u-\frac{\varphi}{\mu}\right)\right\|^{2}+\left\|\partial\left(u-\frac{\varphi}{\mu}\right)\right\|^{2}+\left\|\nabla \cdot\left(\chi_{j} \partial \varphi\right)\right\|_{H^{1}}^{2} \\
& \lesssim\left\|\left(u_{t}, \nabla u, \nabla \varphi\right)\right\|_{H^{1}}^{2}+\|\nabla \phi\|^{2}+\left\|\chi_{j} \partial^{2} \nabla u\right\|^{2}+\left\|\chi_{j} \partial \partial_{y_{3}}\left(\frac{D(\nabla \cdot \varphi)}{D t}\right)\right\|^{2}+\left\|\chi_{j} \partial \nabla(\nabla \cdot \varphi)\right\|^{2} \\
& \quad+\|\nabla u\|_{H^{1}}^{2}\left\|\nabla^{2} u\right\|^{2}+\|\nabla \varphi\|_{H^{2}}^{2}\left\|\nabla^{2} \varphi\right\|_{H^{1}}^{2}+\|\nabla \varphi\|_{H^{2}}^{2}\|\nabla u\|_{H^{2}}^{2} \\
& \lesssim\left\|\left(u_{t}, \nabla u, \nabla \varphi\right)\right\|_{H^{1}}^{2}+\|\nabla \phi\|^{2}+\left\|\chi_{j} \partial^{2} \nabla u\right\|^{2}+\left\|\chi_{j} \partial \partial_{y_{3}}\left(\frac{D(\nabla \cdot \varphi)}{D t}\right)\right\|^{2}+\left\|\chi_{j} \partial \nabla(\nabla \cdot \varphi)\right\|^{2} \\
& \quad+\epsilon\left(\|\nabla u\|_{H^{2}}^{2}+\left\|\nabla^{2} \varphi\right\|_{H^{1}}^{2}\right),
\end{aligned}
$$

where we have estimated

$$
\begin{aligned}
\left\|X_{j} \partial \nabla\left(\frac{D(\nabla \cdot \varphi)}{D t}\right)\right\| & \lesssim\left\|X_{j} \partial^{2}\left(\frac{D(\nabla \cdot \varphi)}{D t}\right)\right\|+\left\|\chi_{j} \partial \partial_{y_{3}}\left(\frac{D(\nabla \cdot \varphi)}{D t}\right)\right\| \\
& \lesssim\left\|X_{j} \partial^{2} \nabla u\right\|+\left\|\chi_{j} \partial \partial_{y_{3}}\left(\frac{D(\nabla \cdot \varphi)}{D t}\right)\right\|
\end{aligned}
$$

and

$$
\left\|\chi_{j} \partial \nabla \phi\right\| \leqslant\left\|\nabla^{2} \phi\right\| \lesssim\|\Delta \phi\|+\|\nabla \phi\| \lesssim\|\nabla \varphi\|+\|\nabla \varphi\|_{L^{\infty}}\|\nabla \varphi\|+\|\nabla \phi\| \lesssim\|\nabla \varphi\|+\|\nabla \phi\| .
$$

Thus we deduce (3.38)-(3.39) from (3.44) and (3.46). Hence, we complete the proof of Lemma 3.9. 
Now we can use the estimates (3.37)-(3.39) in Lemma 3.9 to derive the desired higher-order dissipation estimates for $(u, \varphi)$.

Lemma 3.10. Let $\chi_{j} \in C_{0}^{\infty}\left(\Theta_{j}\right)$ be any fixed function. It holds that

$$
\begin{aligned}
& \frac{d}{d t}\left\|\nabla^{2} \varphi\right\|^{2}+\left\|\nabla^{2}(u, \varphi)\right\|^{2} \lesssim\left\|\left(u_{t}, \nabla u, \nabla \varphi, \nabla \phi, \chi_{0} \nabla^{2} u, \chi_{j} \partial \nabla u, \chi_{j} \partial_{y_{3}}\left(\frac{D(\nabla \cdot \varphi)}{D t}\right)\right)\right\|^{2} \\
& +\left\|\left(\chi_{0} \nabla^{2} \varphi, \chi_{j} \partial \nabla \varphi, \chi_{j} \partial_{y_{3}}(\nabla \cdot \varphi)\right)\right\|^{2} \text {; } \\
& \frac{d}{d t}\left\|\nabla^{3} \varphi\right\|^{2}+\left\|\nabla^{3}(u, \varphi)\right\|^{2} \lesssim\left\|\left(u_{t}, \nabla u, \nabla \varphi, \nabla \phi\right)\right\|_{H^{1}}^{2}+\left\|\left(\chi_{0} \nabla^{3} u, \chi_{j} \partial \nabla^{2} u, \chi_{j} \partial_{y_{3}} \nabla\left(\frac{D(\nabla \cdot \varphi)}{D t}\right)\right)\right\|^{2} \\
& +\left\|\left(\chi_{0} \nabla^{3} \varphi, \chi_{j} \partial \nabla^{2} \varphi, \chi_{j} \partial_{y_{3}} \nabla(\nabla \cdot \varphi)\right)\right\|^{2} \text {; } \\
& \frac{d}{d t}\left\|\chi_{j} \partial \nabla^{2} \varphi\right\|^{2}+\left\|\chi_{j} \partial \nabla^{2}\left(u-\frac{\varphi}{\mu}\right)\right\|^{2} \lesssim\left\|\left(u_{t}, \nabla u, \nabla \varphi\right)\right\|_{H^{1}}^{2}+\left\|\chi_{j}\left(\partial^{2} \nabla u, \partial \partial_{y_{3}}\left(\frac{D(\nabla \cdot \varphi)}{D t}\right), \partial^{2}(\nabla \cdot \varphi), \partial \partial_{y_{3}}(\nabla \cdot \varphi)\right)\right\|^{2} \\
& +\|\nabla \phi\|^{2}+\epsilon\left(\|\nabla u\|_{H^{2}}^{2}+\left\|\nabla^{2} \varphi\right\|_{H^{1}}^{2}\right) .
\end{aligned}
$$

Proof. First, we can establish the following estimates:

$$
\begin{aligned}
& \frac{d}{d t}\left\|\nabla^{2} \varphi\right\|^{2}+\left\|\nabla^{2} \varphi\right\|^{2} \lesssim\left\|\nabla^{2}\left(u-\frac{\varphi}{\mu}\right)\right\|^{2}+\epsilon\|\nabla u\|_{H^{1}}^{2} ; \\
& \frac{d}{d t}\left\|\nabla^{3} \varphi\right\|^{2}+\left\|\nabla^{3} \varphi\right\|^{2} \lesssim\left\|\nabla^{3}\left(u-\frac{\varphi}{\mu}\right)\right\|^{2}+\epsilon\|\nabla u\|_{H^{2}}^{2} ; \\
& \frac{d}{d t}\left\|\chi_{j} \partial \nabla^{2} \varphi\right\|^{2}+\left\|\chi_{j} \partial \nabla^{2} \varphi\right\|^{2} \lesssim\left\|\chi_{j} \partial \nabla^{2}\left(u-\frac{\varphi}{\mu}\right)\right\|^{2}+\epsilon\|\nabla u\|_{H^{2}}^{2} .
\end{aligned}
$$

In fact, applying $\nabla^{2}$ to $(2.8)_{2}$, multiplying it by $\nabla^{2} \varphi$ and integrating over $\Omega$, we have

$$
\begin{aligned}
& \frac{1}{2} \frac{d}{d t} \int_{\Omega}\left|\nabla^{2} \varphi\right|^{2} d x+\frac{1}{\mu} \int_{\Omega}\left|\nabla^{2} \varphi\right|^{2} d x \\
& =-\int_{\Omega} \nabla^{2}\left(u-\frac{\varphi}{\mu}\right) \cdot \nabla^{2} \varphi d x-\int_{\Omega} \nabla^{2}(u \cdot \nabla \varphi) \cdot \nabla^{2} \varphi d x \\
& \lesssim\left\|\nabla^{2}\left(u-\frac{\varphi}{\mu}\right)\right\|\left\|\nabla^{2} \varphi\right\|+\left\|\nabla^{2} u\right\|\|\nabla \varphi\|_{L^{\infty}}\left\|\nabla^{2} \varphi\right\| \\
& +\|\nabla u\|_{L^{3}}\left\|\nabla^{2} \varphi\right\|_{L^{6}}\left\|\nabla^{2} \varphi\right\|+\|u\|_{L^{\infty}}\left\|\nabla^{3} \varphi\right\|\left\|\nabla^{2} \varphi\right\| \\
& \lesssim \epsilon\left(\left\|\nabla^{2} \varphi\right\|^{2}+\|\nabla u\|_{H^{1}}^{2}\right)+\left\|\nabla^{2}\left(u-\frac{\varphi}{\mu}\right)\right\|^{2} .
\end{aligned}
$$

Therefore, we obtain (3.50). Similarly we can prove (3.51) and (3.52).

Next, plugging $(3.50) \times \tilde{\epsilon}(\tilde{\epsilon}>0$ small) into (3.37), we obtain

$$
\frac{d}{d t}\left\|\nabla^{2} \varphi\right\|^{2}+\left\|\nabla^{2}(u, \varphi)\right\|^{2} \lesssim\left\|\left(u_{t}, \nabla u, \nabla \varphi, \nabla \phi, \chi_{0} \nabla^{2} u, \chi_{j} \partial \nabla u, \chi_{j} \partial_{y_{3}}\left(\frac{D(\nabla \cdot \varphi)}{D t}\right)\right)\right\|^{2}+\|\nabla(\nabla \cdot \varphi)\|^{2} .
$$

We estimate

$$
\|\nabla(\nabla \cdot \varphi)\| \lesssim\left\|\left(\chi_{0} \nabla^{2} \varphi, \chi_{j} \partial \nabla \varphi, \chi_{j} \partial_{y_{3}}(\nabla \cdot \varphi)\right)\right\| .
$$

Plugging (3.54) into (3.53), we deduce (3.47). Similarly, we can deduce (3.48) from (3.51) and (3.38), as well as (3.49) from (3.52) and (3.39).

\section{Proof of Theorem 2.1}

In this section, we will establish the a priori estimates based on the lemmas in Section 3. Once we have the a prior estimates, the proof of Theorem 2.1 is natural. 
Table 1: List of Energy Estimates

\begin{tabular}{llll}
\hline Lemma & Energy $\mathcal{E}(t)$ & Dissipation $\mathcal{D}(t)$ & Key terms in $\mathcal{B}(t)$ \\
\hline 3.1 & $u, \nabla \varphi, \nabla \phi$ & $\nabla u$ & - \\
3.2 & $u_{t}, \nabla \varphi_{t}, \nabla \phi_{t}$ & $\nabla u_{t}$ & - \\
3.3 & - & $\nabla(\varphi, \phi)$ & $\nabla u_{t}, \nabla u$ \\
3.4 & $\nabla u$ & $u_{t}$ & - \\
3.5 & $\chi_{0}(\nabla u, \Delta \varphi, \Delta \phi)$ & $\chi_{0} \nabla^{2} u$ & $\nabla u$ \\
& $\chi_{0}\left(\nabla^{2} u, \nabla \Delta \varphi, \nabla \Delta \phi\right)$ & $\chi_{0} \nabla^{3} u$ & $\nabla^{2} u$ \\
& - & $\chi_{0} \nabla^{2}(\varphi, \phi)$ & $\chi_{0} \nabla^{2} u, \nabla \varphi$ \\
3.6 & - & $\chi_{0} \nabla^{3}(\varphi, \phi)$ & $\chi_{0} \nabla^{3} u, \nabla^{2} \varphi$ \\
& $\chi_{j} \partial(u, \nabla \varphi, \nabla \phi)$ & $\chi_{j} \partial \nabla u$ & $\nabla u$ \\
& $\chi_{j} \partial^{2}(u, \nabla \varphi, \nabla \phi)$ & $\chi_{j} \partial^{2} \nabla u$ & $\nabla^{2} u$ \\
3.7 & - & $\chi_{j} \partial(\nabla \varphi, \nabla \phi)$ & $\chi_{j} \partial \nabla u$ \\
3.8 & - & $\chi_{j} \partial^{2}(\nabla \varphi, \nabla \phi)$ & $\chi_{j} \partial^{2} \nabla u$ \\
& $\chi_{j} \partial \nabla u$ & $\chi_{j} \partial u_{t}$ & $\nabla^{2} u, \nabla^{3} \varphi$ \\
& $\chi_{j} \partial_{y_{3}}(\nabla \cdot \varphi)$ & $\chi_{j} \partial_{y_{3}}\left(\frac{D(\nabla \cdot \varphi)}{D t}\right), \chi_{j} \partial_{y_{3}}(\nabla \cdot \varphi)$ & $\chi_{j}(\partial \nabla u, \partial \nabla \varphi)$ \\
3.9 & $\chi_{j} \partial \partial_{y_{3}}(\nabla \cdot \varphi)$ & $\chi_{j} \partial \partial_{y_{3}}\left(\frac{D(\nabla \cdot \varphi)}{D t}\right), \chi_{j} \partial \partial_{y_{3}}(\nabla \cdot \varphi)$ & $\chi_{j}\left(\partial^{2} \nabla u, \partial^{2} \nabla \varphi\right)$ \\
3.10 & $\chi_{j} \partial_{y_{3}}^{2}(\nabla \cdot \varphi)$ & $\chi_{j} \partial_{y_{3}}^{2}\left(\frac{D(\nabla \cdot \varphi)}{D t}\right), \chi_{j} \partial_{y_{3}}^{2}(\nabla \cdot \varphi)$ & $\chi_{j}\left(\partial \partial_{y_{3}} \nabla u, \partial \partial_{y_{3}} \nabla \varphi\right)$ \\
& - & - & - \\
& $\nabla^{2} \varphi$ & $\nabla^{2}(u, \varphi)$ & $\chi_{j} \partial_{y_{3}}\left(\frac{D(\nabla \cdot \varphi)}{D t}\right), \chi_{j} \partial_{y_{3}}(\nabla \cdot \varphi)$ \\
& $\chi_{j} \partial \nabla^{2} \varphi$ & $\chi_{j} \partial \nabla \chi_{j} \partial \nabla \varphi$ \\
& $\nabla^{3} \varphi$ & $\chi_{j}(u, \varphi)$ & $\chi_{j} \partial \partial_{y_{3}}\left(\frac{D(\nabla \cdot \varphi)}{D t}\right)$ \\
& & & $\chi_{j} \partial^{2}(\nabla \cdot \varphi), \chi_{j} \partial \partial_{y_{3}}(\nabla \cdot \varphi)$ \\
& & $\chi_{j} \partial_{y_{3}} \nabla\left(\frac{D(\nabla \cdot \varphi)}{D t}\right), \chi_{j} \partial_{y_{3}} \nabla(\nabla \cdot \varphi)$ & $\chi_{j} \partial \nabla^{2} u, \chi_{j} \partial \nabla \nabla^{2} \varphi$ \\
\hline
\end{tabular}

Note that the energy estimates obtained in Section 3 are all of form

$$
\frac{d}{d t} \mathcal{E}(t)+\mathcal{D}(t) \lesssim \mathcal{B}(t)+\epsilon \mathcal{S}(t), \quad \epsilon \ll 1,
$$

where $\mathcal{B}(t)$ contains some bad large terms. For clarity, we make a table to illustrate that the bad terms $\mathcal{B}(t)$ appeared in some row can be controlled by the dissipation $\mathcal{D}(t)$ located in other rows after multiplying them by a small constant. For simplicity, we omit the transformation of the domains of integration between $x$-domain and $y$-domain since their equivalence in norms due to (3.20)-(3.21). We keep in mind that the summation $\sum_{j=1}^{N}$ will be taken once $\chi_{j}$ occurs in the following.

Now, let us do the derivations in detail. Let $\tilde{\epsilon}>0$ be any small constant, which can be different from line to line. Applying (3.8) $\times \tilde{\epsilon}+(3.2)+(3.4)$, we have

$$
\frac{d}{d t}\left\|\left(u, \nabla \cdot \varphi, \nabla \varphi, \nabla \phi, u_{t}, \nabla \cdot \varphi_{t}, \nabla \varphi_{t}, \nabla \phi_{t}\right)\right\|^{2}+\left\|\left(\nabla u, \nabla \varphi, \nabla \phi, \nabla u_{t}\right)\right\|^{2} \leqslant \epsilon\left\|\nabla^{2}(u, \varphi)\right\|^{2} .
$$

Adding (3.10) $\times \tilde{\epsilon}$ to (4.1), we have

$$
\frac{d}{d t}\left\|\left(u, \nabla u, \nabla \cdot u, \nabla \cdot \varphi, \nabla \varphi, \nabla \phi, u_{t}, \nabla \cdot \varphi_{t}, \nabla \varphi_{t}, \nabla \phi_{t}\right)\right\|^{2}+\left\|\left(\nabla u, \nabla \varphi, \nabla \phi, u_{t}, \nabla u_{t}\right)\right\|^{2} \leqslant \epsilon\left\|\nabla^{2}(u, \varphi)\right\|^{2} .
$$

Applying [(3.15) $+(3.16)] \times \tilde{\epsilon}^{2}+[(3.13)+(3.14)] \times \tilde{\epsilon}+(4.2)$, by choosing $\tilde{\epsilon}>0$ to be small enough so that the righthand side terms $\left\|\chi_{0}\left(\nabla^{2} u, \nabla^{3} u\right)\right\|^{2}$ in [(3.15) + (3.16)] can be absorbed by the left-hand side of [(3.13) + (3.14)], we have

$$
\begin{aligned}
& \frac{d}{d t}\left\|\left(u, u_{t}, \nabla u, \nabla \cdot u, \chi_{0} \nabla u, \chi_{0} \nabla^{2} u\right)\right\|^{2} \\
& \quad+\frac{d}{d t}\left\|\left(\nabla \cdot \varphi, \nabla \varphi, \nabla \cdot \varphi_{t}, \nabla \varphi_{t}, \chi_{0} \nabla \nabla \cdot \varphi, \chi_{0} \Delta \varphi, \chi_{0} \nabla^{2} \nabla \cdot \varphi, \chi_{0} \nabla \Delta \varphi\right)\right\|^{2} \\
& \quad+\frac{d}{d t}\left\|\left(\nabla \phi, \nabla \phi_{t}, \chi_{0} \Delta \phi, \chi_{0} \nabla \Delta \phi\right)\right\|^{2}
\end{aligned}
$$




$$
\begin{aligned}
& +\left\|\left(\nabla u, \nabla \varphi, \nabla \phi, u_{t}, \nabla u_{t}\right)\right\|^{2}+\left\|\chi_{0}\left(\nabla^{2} u, \nabla^{3} u, \nabla^{2} \varphi, \nabla^{2} \phi, \nabla^{3} \varphi, \nabla^{3} \phi\right)\right\|^{2} \\
& \quad \leqslant \tilde{\epsilon}\left\|\nabla^{2}(u, \varphi)\right\|^{2}+\epsilon\left\|\nabla^{2}(u, \varphi)\right\|_{H^{1}}^{2} .
\end{aligned}
$$

Applying $[(3.25)+(3.26)] \times \tilde{\epsilon}^{2}+[(3.23)+(3.24)] \times \tilde{\epsilon}+(4.3)$, by choosing $\tilde{\epsilon}>0$ to be small enough so that the righthand side terms $\left\|\chi_{j}\left(\partial \nabla u, \partial^{2} \nabla u\right)\right\|^{2}$ in [(3.25) + (3.26)] can be absorbed by the left-hand side of [(3.23) + (3.24)], we have

$$
\begin{aligned}
& \frac{d}{d t}\left\|\left(u, u_{t}, \nabla u, \nabla \cdot u, \chi_{0} \nabla u, \chi_{0} \nabla^{2} u\right)\right\|^{2} \\
& +\frac{d}{d t}\left\|\left(\nabla \cdot \varphi, \nabla \varphi, \nabla \cdot \varphi_{t}, \nabla \varphi_{t}, \chi_{0} \nabla \nabla \cdot \varphi, \chi_{0} \Delta \varphi, \chi_{0} \nabla^{2} \nabla \cdot \varphi, \chi_{0} \nabla \Delta \varphi\right)\right\|^{2} \\
& +\frac{d}{d t}\left\|\left(\nabla \phi, \nabla \phi_{t}, \chi_{0} \Delta \phi, \chi_{0} \nabla \Delta \phi\right)\right\|^{2} \\
& +\frac{d}{d t}\left\|\chi_{j}\left(\partial u, \partial^{2} u, \partial \nabla \cdot \varphi, \partial^{2} \nabla \cdot \varphi, \partial \nabla \varphi, \partial^{2} \nabla \varphi, \partial \nabla \phi, \partial^{2} \nabla \phi\right)\right\|^{2} \\
& \quad+\left\|\left(\nabla u, \nabla \varphi, \nabla \phi, u_{t}, \nabla u_{t}\right)\right\|^{2}+\left\|\chi_{0}\left(\nabla^{2} u, \nabla^{3} u, \nabla^{2} \varphi, \nabla^{3} \varphi, \nabla^{2} \phi, \nabla^{3} \phi\right)\right\|^{2} \\
& \quad+\left\|\chi_{j}\left(\partial \nabla u, \partial^{2} \nabla u, \partial \nabla \varphi, \partial^{2} \nabla \varphi, \partial \nabla \phi, \partial^{2} \nabla \phi\right)\right\|^{2} \\
& \quad \leqslant \tilde{\epsilon}\left\|\nabla^{2}(u, \varphi)\right\|^{2}+\epsilon\left\|\nabla^{2}(u, \varphi)\right\|_{H^{1}}^{2} .
\end{aligned}
$$

Applying (3.47) $\times \tilde{\epsilon}^{\frac{1}{2}}+(3.28) \times \tilde{\epsilon}^{\frac{1}{4}}+(4.4)$, by choosing $\tilde{\epsilon}>0$ to be small enough so that the right-hand side terms $\left\|\chi_{j} \partial_{y_{3}}\left(\frac{D(\nabla \cdot \varphi)}{D t}\right)\right\|^{2},\left\|\chi_{j} \partial_{y_{3}}(\nabla \cdot \varphi)\right\|^{2}$ in (3.47) can be absorbed by the left-hand side of (3.28), we have

$$
\begin{aligned}
& \frac{d}{d t}\left\|\left(u, u_{t}, \nabla u, \nabla \cdot u, \chi_{0} \nabla u, \chi_{0} \nabla^{2} u\right)\right\|^{2} \\
& +\frac{d}{d t}\left\|\left(\nabla \cdot \varphi, \nabla \varphi, \nabla \cdot \varphi_{t}, \nabla \varphi_{t}, \chi_{0} \nabla \nabla \cdot \varphi, \chi_{0} \Delta \varphi, \chi_{0} \nabla^{2} \nabla \cdot \varphi, \chi_{0} \nabla \Delta \varphi, \nabla^{2} \varphi\right)\right\|^{2} \\
& +\frac{d}{d t}\left\|\left(\nabla \phi, \nabla \phi_{t}, \chi_{0} \Delta \phi, \chi_{0} \nabla \Delta \phi\right)\right\|^{2} \\
& +\frac{d}{d t}\left\|\chi_{j}\left(\partial u, \partial^{2} u, \partial \nabla \cdot \varphi, \partial^{2} \nabla \cdot \varphi, \partial \nabla \varphi, \partial^{2} \nabla \varphi, \partial \nabla \phi, \partial^{2} \nabla \phi\right)\right\|^{2}+\frac{d}{d t}\left\|\chi_{j} \partial_{y_{3}}(\nabla \cdot \varphi)\right\|^{2} \\
& \quad+\left\|\left(\nabla u, \nabla \varphi, \nabla \phi, u_{t}, \nabla u_{t}\right)\right\|^{2}+\left\|\chi_{0}\left(\nabla^{2} u, \nabla^{3} u, \nabla^{2} \varphi, \nabla^{3} \varphi, \nabla^{2} \phi, \nabla^{3} \phi\right)\right\|^{2} \\
& \quad+\left\|\chi_{j}\left(\partial \nabla u, \partial^{2} \nabla u, \partial \nabla \varphi, \partial^{2} \nabla \varphi, \partial \nabla \phi, \partial^{2} \nabla \phi\right)\right\|^{2} \\
& \quad+\left\|\chi_{j} \partial_{y_{3}}\left(\frac{D(\nabla \cdot \varphi)}{D t}\right)\right\|^{2}+\left\|\chi_{j} \partial_{y_{3}}(\nabla \cdot \varphi)\right\|^{2}+\left\|\left(\nabla^{2} \varphi, \nabla^{2} u\right)\right\|^{2} \leqslant \epsilon\left\|\nabla^{3}(u, \varphi)\right\|^{2} .
\end{aligned}
$$

Applying (3.29) ${ }_{\kappa=1} \times \tilde{\epsilon}+(3.49) \times \tilde{\epsilon}^{2}+(3.29)_{\kappa=0} \times \tilde{\epsilon}^{3}+(4.5)$, by choosing $\tilde{\epsilon}>0$ to be small enough so that: the right-hand side terms $\left\|\chi_{j}\left(\partial^{2} \nabla u, \partial^{2} \nabla \varphi\right)\right\|^{2}$ in (3.29) ${ }_{\kappa=1}$ can be absorbed by the left-hand side of (4.5), the righthand side terms $\left\|\chi_{j}\left(\partial \partial_{y_{3}}\left(\frac{D(\nabla \cdot \varphi)}{D t}\right), \partial \partial_{y_{3}}(\nabla \cdot \varphi)\right)\right\|^{2}$ in (3.49) can be absorbed by the left-hand side of (3.29) $)_{\kappa=1}$, the right-hand side terms $\left\|\chi_{j}\left(\partial \partial_{y_{3}} \nabla u, \partial \partial_{y_{3}} \nabla \varphi\right)\right\|^{2}$ of (3.29) ${ }_{\kappa=0}$ can be absorbed by the left-hand side of (3.49), we have

$$
\begin{aligned}
& \frac{d}{d t}\left\|\left(u, u_{t}, \nabla u, \nabla \cdot u, \chi_{0} \nabla u, \chi_{0} \nabla^{2} u\right)\right\|^{2} \\
& +\frac{d}{d t}\left\|\left(\nabla \cdot \varphi, \nabla \varphi, \nabla \cdot \varphi_{t}, \nabla \varphi_{t}, \chi_{0} \nabla \nabla \cdot \varphi, \chi_{0} \Delta \varphi, \chi_{0} \nabla^{2} \nabla \cdot \varphi, \chi_{0} \nabla \Delta \varphi, \nabla^{2} \varphi\right)\right\|^{2} \\
& +\frac{d}{d t}\left\|\left(\nabla \phi, \nabla \phi_{t}, \chi_{0} \Delta \phi, \chi_{0} \nabla \Delta \phi\right)\right\|^{2} \\
& +\frac{d}{d t}\left\|\chi_{j}\left(\partial u, \partial^{2} u, \partial \nabla \cdot \varphi, \partial^{2} \nabla \cdot \varphi, \partial \nabla \varphi, \partial^{2} \nabla \varphi, \partial \nabla \phi, \partial^{2} \nabla \phi\right)\right\|^{2} \\
& +\frac{d}{d t}\left\|\chi_{j}\left[\partial_{y_{3}}(\nabla \cdot \varphi), \partial \partial_{y_{3}}(\nabla \cdot \varphi), \partial_{y_{3}}^{2}(\nabla \cdot \varphi), \partial \nabla^{2} \varphi\right]\right\|^{2} \\
& \quad+\left\|\left(\nabla u, \nabla \varphi, \nabla \phi, u t, \nabla u_{t}\right)\right\|^{2}+\left\|\chi_{0}\left(\nabla^{2} u, \nabla^{3} u, \nabla^{2} \varphi, \nabla^{3} \varphi, \nabla^{2} \phi, \nabla^{3} \phi\right)\right\|^{2} \\
& \quad+\left\|\chi_{j}\left(\partial \nabla u, \partial^{2} \nabla u, \partial \nabla \varphi, \partial^{2} \nabla \varphi, \partial \nabla \phi, \partial^{2} \nabla \phi\right)\right\|^{2} \\
& \quad+\left\|\chi_{j} \partial_{y_{3}}\left(\frac{D(\nabla \cdot \varphi)}{D t}\right)\right\|^{2}+\left\|\chi_{j} \partial_{y_{3}}(\nabla \cdot \varphi)\right\|^{2}+\left\|\left(\nabla^{2} \varphi, \nabla^{2} u\right)\right\|^{2} \\
& \quad+\left\|\chi_{j} \partial \partial_{y_{3}}\left(\frac{D(\nabla \cdot \varphi)}{D t}\right)\right\|^{2}+\left\|\chi_{j} \partial \partial_{y_{3}}(\nabla \cdot \varphi)\right\|^{2}+\left\|\chi_{j} \partial \nabla^{2}(u, \varphi)\right\|^{2}
\end{aligned}
$$




$$
+\left\|\chi_{j} \partial_{y_{3}}^{2}\left(\frac{D(\nabla \cdot \varphi)}{D t}\right)\right\|^{2}+\left\|\chi_{j} \partial_{y_{3}}^{2}(\nabla \cdot \varphi)\right\|^{2} \leqslant \epsilon\left\|\nabla^{3}(u, \varphi)\right\|^{2} .
$$

Applying (3.48) × $\tilde{\epsilon}+(4.6)$, we have

$$
\begin{aligned}
\frac{d}{d t}\left\|\left(u, u_{t}, \nabla u, \nabla \cdot u, \chi_{0} \nabla u, \chi_{0} \nabla^{2} u\right)\right\|^{2} \\
+\frac{d}{d t}\left\|\left(\nabla \cdot \varphi, \nabla \varphi, \nabla \cdot \varphi_{t}, \nabla \varphi_{t}, \chi_{0} \nabla \nabla \cdot \varphi, \chi_{0} \Delta \varphi, \chi_{0} \nabla^{2} \nabla \cdot \varphi, \chi_{0} \nabla \Delta \varphi, \nabla^{2} \varphi, \nabla^{3} \varphi\right)\right\|^{2} \\
+\frac{d}{d t}\left\|\left(\nabla \phi, \nabla \phi_{t}, \chi_{0} \Delta \phi, \chi_{0} \nabla \Delta \phi\right)\right\|^{2} \\
+\frac{d}{d t}\left\|\chi_{j}\left(\partial u, \partial^{2} u, \partial \nabla \cdot \varphi, \partial^{2} \nabla \cdot \varphi, \partial \nabla \varphi, \partial^{2} \nabla \varphi, \partial \nabla \phi, \partial^{2} \nabla \phi\right)\right\|^{2} \\
+\frac{d}{d t}\left\|\chi_{j}\left[\partial_{y_{3}}(\nabla \cdot \varphi), \partial \partial_{y_{3}}(\nabla \cdot \varphi), \partial_{y_{3}}^{2}(\nabla \cdot \varphi), \partial \nabla^{2} \varphi\right]\right\|^{2} \\
\quad+\left\|\left(\nabla u, \nabla \varphi, \nabla \phi, u_{t}, \nabla u_{t}\right)\right\|^{2}+\left\|\chi_{0}\left(\nabla^{2} u, \nabla^{3} u, \nabla^{2} \varphi, \nabla^{3} \varphi, \nabla^{2} \phi, \nabla^{3} \phi\right)\right\|^{2} \\
\quad+\left\|\chi_{j}\left(\partial \nabla u, \partial^{2} \nabla u, \partial \nabla \varphi, \partial^{2} \nabla \varphi, \partial \nabla \phi, \partial^{2} \nabla \phi\right)\right\|^{2} \\
\quad+\left\|\chi_{j} \partial_{y_{3}}\left(\frac{D(\nabla \cdot \varphi)}{D t}\right)\right\|^{2}+\left\|\chi_{j} \partial_{y_{3}}(\nabla \cdot \varphi)\right\|^{2}+\left\|\left(\nabla^{2} u, \nabla^{2} \varphi\right)\right\|^{2}+\left\|\left(\nabla^{3} u, \nabla^{3} \varphi\right)\right\|^{2} \\
\quad+\left\|\chi_{j} \partial \partial_{y_{3}}\left(\frac{D(\nabla \cdot \varphi)}{D t}\right)\right\|^{2}+\left\|\chi_{j} \partial \partial_{y_{3}}(\nabla \cdot \varphi)\right\|^{2}+\left\|\chi_{j} \partial \nabla^{2}(u, \varphi)\right\|^{2} \\
\quad+\left\|\chi_{j} \partial_{y_{3}}^{2}\left(\frac{D(\nabla \cdot \varphi)}{D t}\right)\right\|^{2}+\left\|\chi_{j} \partial_{y_{3}}^{2}(\nabla \cdot \varphi)\right\|^{2} \leqslant 0 .
\end{aligned}
$$

Applying (3.27) $\times \tilde{\epsilon}+(4.7)$, we have

$$
\begin{aligned}
\frac{d}{d t}\left\|\left(u, \nabla u, \nabla \cdot u, u_{t}\right)\right\|^{2}+\frac{d}{d t}\left\|\chi_{0}\left(\nabla u, \nabla^{2} u\right)\right\|^{2}+\frac{d}{d t}\left\|\chi_{j}\left(\partial u, \partial^{2} u, \partial \nabla u, \partial \nabla \cdot u\right)\right\|^{2} \\
+\frac{d}{d t}\left\|\left(\nabla \cdot \varphi, \nabla \varphi, \nabla^{2} \varphi, \nabla^{3} \varphi, \nabla \cdot \varphi_{t}, \nabla \varphi \varphi_{t}\right)\right\|^{2}+\frac{d}{d t}\left\|\chi_{0}\left(\nabla \nabla \cdot \varphi, \Delta \varphi, \nabla^{2} \nabla \cdot \varphi, \nabla \Delta \varphi\right)\right\|^{2} \\
+\frac{d}{d t}\left\|\chi_{j}\left[\partial_{y_{3}}(\nabla \cdot \varphi), \partial(\nabla \cdot \varphi), \partial \nabla \varphi, \partial^{2}(\nabla \cdot \varphi), \partial^{2} \nabla \varphi, \partial \partial_{y_{3}}(\nabla \cdot \varphi), \partial_{y_{3}}^{2}(\nabla \cdot \varphi), \partial \nabla^{2} \varphi\right]\right\|^{2} \\
+\frac{d}{d t}\left\|\left(\nabla \phi, \nabla \phi_{t}\right)\right\|^{2}+\frac{d}{d t}\left\|\chi_{0}(\Delta \phi, \nabla \Delta \phi)\right\|^{2}+\frac{d}{d t}\left\|\chi_{j}\left(\partial \nabla \phi, \partial^{2} \nabla \phi\right)\right\|^{2} \\
\quad+\left\|\left(\nabla u, \nabla^{2} u, \nabla^{3} u, u_{t}, \nabla u_{t}\right)\right\|^{2}+\left\|\chi_{0}\left(\nabla^{2} u, \nabla^{3} u\right)\right\|^{2}+\left\|\chi_{j}\left(\partial \nabla u, \partial^{2} \nabla u, \partial \nabla^{2} u, \partial u_{t}\right)\right\|^{2} \\
\quad+\left\|\left(\nabla \varphi, \nabla^{2} \varphi, \nabla^{3} \varphi\right)\right\|^{2}+\left\|\chi_{0}\left(\nabla^{2} \varphi, \nabla^{3} \varphi\right)\right\|^{2} \\
\quad+\left\|\chi_{j}\left[\partial \nabla \varphi, \partial^{2} \nabla \varphi, \partial \nabla^{2} \varphi, \partial_{y_{3}}(\nabla \cdot \varphi), \partial \partial_{y_{3}}(\nabla \cdot \varphi), \partial_{y_{3}}^{2}(\nabla \cdot \varphi)\right]\right\|^{2} \\
\quad+\|\nabla \phi\|^{2}+\left\|\chi_{0}\left(\nabla^{2} \phi, \nabla^{3} \phi\right)\right\|^{2}+\left\|\chi_{j}\left(\partial \nabla \phi, \partial^{2} \nabla \phi\right)\right\|^{2} \\
\quad+\left\|\chi_{i}\left[\partial_{y_{3}}\left(\frac{D(\nabla \cdot \varphi)}{D t}\right), \partial \partial_{y_{3}}\left(\frac{D(\nabla \cdot \varphi)}{D t}\right), \partial_{y_{3}}^{2}\left(\frac{D(\nabla \cdot \varphi)}{D t}\right)\right]\right\|^{2} \leqslant 0 .
\end{aligned}
$$

We collect all the terms under the time derivative in (4.8) and then denote all of them by $Y(t)$. Then (4.8) becomes

$$
\frac{d}{d t} Y(t)+C\left(Y(t)+\left\|X_{i} \partial_{y_{3}}^{2} u\right\|^{2}+\left\|\nabla^{3} u\right\|^{2}\right) \leqslant 0 .
$$

By Poincarê's inequality (cf. Lemma A.1), we easily check that

$$
Y(t) \sim\|u\|_{H^{1}}^{2}+\left\|\chi_{0} \nabla^{2} u\right\|^{2}+\left\|\chi_{j} \partial \nabla u\right\|^{2}+\|\varphi\|_{H^{3}}^{2}+\|\nabla \phi\|_{H^{2}}^{2}+\left\|\left(u_{t}, \nabla \varphi_{t}, \nabla \phi_{t}\right)\right\|^{2} .
$$

Applying Gronwall's inequality to (4.9), we obtain

$$
Y(t) e^{C t}+C \int_{0}^{t} e^{C s}\left(\left\|X_{j} \partial_{y_{3}}^{2} u\right\|^{2}+\left\|\nabla^{3} u\right\|^{2}\right) d s \leqslant Y(0) .
$$

By $(2.8)_{1}$, we easily estimate

$$
\left\|\chi_{j} \partial_{y_{3}}^{2} u\right\|^{2} \lesssim\left\|\left(\chi_{j} \partial \nabla u, \chi_{0} \nabla^{2} u, u_{t}, \nabla \nabla \cdot \varphi, \Delta \varphi, \nabla \phi, R_{1}\right)\right\|^{2} \lesssim Y(t) .
$$


Combining (4.10)-(4.12) with (3.1), by (2.8), there exists a functional $\mathcal{H}(t)$ satisfying

$$
\mathcal{H}(t) \sim\|(\rho-1, u, \mathbb{F}-\mathbb{I})\|_{H^{2}}^{2}+\|\varphi\|_{H^{3}}^{2}+\|\nabla \phi\|_{H^{2}}^{2}+\left\|\left(\rho_{t}, u_{t}, \mathbb{F}_{t}, \nabla \varphi_{t}, \nabla \phi_{t}\right)\right\|^{2}
$$

such that

$$
\mathcal{H}(t) e^{C t}+C \int_{0}^{t} e^{C s}\left(\left\|\chi_{j} \partial_{y_{3}}^{2} u\right\|^{2}+\left\|\nabla^{3} u\right\|^{2}\right) d s \lesssim \mathcal{H}(0) \lesssim\left\|\left(\rho_{0}-1, u_{0}, \mathbb{F}_{0}-\mathbb{I}\right)\right\|_{H^{2}}
$$

From the above, we have proved the following a priori estimates:

Proposition 4.1 (A priori estimates). Let $T>0$. Assume that for sufficiently small $\epsilon>0$,

$$
\sup _{t \in[0, T]}\|(\rho-1, u, \mathbb{F}-\mathbb{I})(t)\|_{H^{2}}<\epsilon .
$$

Then we have for any $t \in[0, T]$ and some $\alpha>0$,

$$
\|(\rho-1, u, \mathbb{F}-\mathbb{I}, \nabla \phi)(t)\|_{H^{2}}+\left\|\left(\rho_{t}, u_{t}, \mathbb{F}_{t}, \nabla \phi_{t}\right)(t)\right\| \leqslant C_{2}\left\|\left(\rho_{0}-1, u_{0}, \mathbb{F}_{0}-\mathbb{I}\right)\right\|_{H^{2}} e^{-\alpha t},
$$

where $C_{2}>1$ is some fixed constant.

Then the local solution given in Proposition 3.1 can be extended to the global one by combining the a priori estimates given in Proposition 4.1 with a standard continuous argument, cf. [35]. The exponential decay rate (2.12) follows from (4.13). Hence, we complete the proof of Theorem 2.1.

\section{A Appendix}

In the appendix, we list some useful lemmas which are frequently used in previous sections. First, we recall the Poincaré's inequality:

Lemma A.1. Let $\Omega$ be a bounded, connected, open subset of $\mathbb{R}^{n}$, with a $C^{1}$ boundary $\partial \Omega$. Assume $1 \leqslant p \leqslant \infty$. (1) If $u \in W_{0}^{1, p}(\Omega)$, then

$$
\|u\|_{L^{p}(\Omega)} \leqslant C\|\nabla u\|_{L^{p}(\Omega)}
$$

(2) If $u \in W^{1, p}(\Omega)$, denoting the average of $u$ over $\Omega$ by $u_{\Omega}=\frac{1}{|\Omega|} \int_{\Omega} u d x$, then

$$
\left\|u-u_{\Omega}\right\|_{L^{p}(\Omega)} \leqslant C\|\nabla u\|_{L^{p}(\Omega)} .
$$

The above constant $C>0$ depends only on $n, p$ and $\Omega$.

Proof. The detailed proof can be found in [6].

Then we recall the classical Gagliardo-Nirenberg-Sobolev inequality on a bounded domain.

Lemma A.2. Let $\Omega$ be a bounded domain of $\mathbb{R}^{n}$ with a $C^{m}$ boundary $\partial \Omega$. Assume $u \in L^{q}(\Omega)$ and $\nabla^{m} u \in L^{p}(\Omega)$ with $1 \leqslant p, q \leqslant \infty$ and $1 \leqslant m \in \mathbb{N}$. Then we have for $k \in\{0,1,2, \ldots, m-1\}$,

$$
\left\|\nabla^{k} u\right\|_{L^{r}(\Omega)} \leqslant C_{1}\left\|\nabla^{m} u\right\|_{L^{p}(\Omega)}^{\alpha}\|u\|_{L^{q}(\Omega)}^{1-\alpha}+C_{2}\|u\|_{L^{q}(\Omega)},
$$

where

$$
\frac{1}{r}=\frac{k}{n}+\alpha\left(\frac{1}{p}-\frac{m}{n}\right)+(1-\alpha) \frac{1}{q}
$$


with

$$
\begin{cases}\frac{k}{m} \leqslant \alpha<1, & \text { if } 1<p<\infty \text { and } m-k-\frac{n}{p} \in \mathbb{N} \cup\{0\}, \\ \frac{k}{m} \leqslant \alpha \leqslant 1, & \text { otherwise. }\end{cases}
$$

The above two positive constants $C_{1}$ and $C_{2}$ depend only on $n, m, k, p, q, \alpha$ and $\Omega$.

A special case: If $u \in W^{m, p}(\Omega) \cap L^{q}(\Omega)$, then we have

$$
\left\|\nabla^{k} u\right\|_{L^{r}(\Omega)} \leqslant C\|u\|_{W^{m, p}(\Omega)}^{\alpha}\|u\|_{L^{q}(\Omega)}^{1-\alpha} .
$$

Proof. See [38].

Next, we give some important time-invariant relations for the density $\rho$ and the deformation gradient $\mathbb{F}$.

Lemma A.3. If the initial data $\left(\rho_{0}, \mathbb{F}_{0}\right)$ satisfy

$$
\nabla \cdot\left(\rho_{0} \mathbb{F}_{0}^{T}\right)=0, \quad \mathbb{F}_{0}^{l k} \nabla_{l} \mathbb{F}_{0}^{i j}-\mathbb{F}_{0}^{l j} \nabla_{l} \mathbb{F}_{0}^{i k}=0, \quad \rho_{0} \operatorname{det} \mathbb{F}_{0}=1,
$$

then $\rho$ and $\mathbb{F}$ in (1.6) shall satisfy

$$
\nabla \cdot\left(\rho \mathbb{F}^{T}\right)=0, \quad \mathbb{F}^{l k} \nabla_{l} \mathbb{F}^{i j}-\mathbb{F}^{l j} \nabla_{l} \mathbb{F}^{i k}=0, \quad \rho \operatorname{det} \mathbb{F}=1 .
$$

Proof. The proof can be found in [41].

Then, we shall give the regularity estimates for the Stokes problem:

$$
\left\{\begin{array}{l}
\nabla \cdot v=h \\
-\Delta v+\nabla q=g \\
\left.v\right|_{\partial \Omega}=a
\end{array}\right.
$$

Lemma A.4. For the Stokes problem (A.1) on a bounded region $\Omega$ with $\partial \Omega \in C^{3}$, we have

$$
\left\|\nabla^{k+2} v\right\|+\left\|\nabla^{k+1} q\right\| \leqslant C\left(\|h\|_{H^{k+1}(\Omega)}+\|g\|_{H^{k}(\Omega)}+\|a\|_{H^{k+\frac{3}{2}(\partial \Omega)}}\right)
$$

for $k=0,1$.

Proof. Refer to [48].

Acknowledgements: Wenpei Wu would like to thank Professor Guochun Wu for several helpful discussions on this topic.

Yong Wang was partially supported by Guangdong Provincial Pearl River Talents Program (No. 2017GC010407), Guangdong Province Basic and Applied Basic Research Fund (Nos. 2021A1515010235 and 2020B1515310002), Guangzhou City Basic and Applied Basic Research Fund (No. 202102020436), the NSF of China (No. 11701264) and Science and Technology Program of Guangzhou (No. 2019050001).

Conflict of Interest: The authors declare that they have no conflict of interest.

\section{References}

[1] H. W. Alt. The entropy principle for interfaces. Fluids and solids. Adv. Math. Sci. Appl., 19(2009), no.2, 585-663.

[2] Q. Y. Bie, Q. R. Wang, and Z. A. Yao. Optimal decay rate for the compressible Navier-Stokes-Poisson system in the critical $L^{p}$ framework. J. Differential Equations, 263(2017), no.12, 8391-8417.

[3] Q. Chen and G. C. Wu. The 3D compressible viscoelastic fluid in a bounded domain. Commun. Math. Sci., 16(2018), no.5, 1303-1323. 
[4] Y. M. Chen and P. Zhang. The global existence of small solutions to the incompressible viscoelastic fluid system in 2 and 3 space dimensions. Comm. Partial Differential Equations, 31(2006), no.10-12, 1793-1810.

[5] M. P. do Carmo. Differential Geometry of Curves \& Surfaces. Dover Publications, Inc., Mineola, NY, 2016. Revised \& updated second edition of [ MR0394451].

[6] L. C. Evans. Partial Differential Equations, volume 19 of Graduate Studies in Mathematics. American Mathematical Society, Providence, RI, second edition, 2010.

[7] Y. Guo and Y. J. Wang. Decay of dissipative equations and negative Sobolev spaces. Comm. Partial Differential Equations, 37(2012), no.12, 2165-2208.

[8] C. C. Hao and H. L. Li. Global existence for compressible Navier-Stokes-Poisson equations in three and higher dimensions. J. Differential Equations, 246(2009), no.12, 4791-4812.

[9] D. Hoff and K. Zumbrun. Multi-dimensional diffusion waves for the Navier-Stokes equations of compressible flow. Indiana Univ. Math. J., 44(1995), no.2, 603-676.

[10] L. Hsiao and H. L. Li. Compressible Navier-Stokes-Poisson equations. Acta Math. Sci. Ser. B (Engl. Ed.), 30(2010), no.6, 1937-1948.

[11] X. P. Hu. Global existence of weak solutions to two dimensional compressible viscoelastic flows. J. Differential Equations, 265(2018), no.7, 3130-3167.

[12] X. P. Hu and F. H. Lin. Global solutions of two-dimensional incompressible viscoelastic flows with discontinuous initial data. Comm. Pure Appl. Math., 69(2016), no.2, 372-404.

[13] X. P. Hu and D. H. Wang. Local strong solution to the compressible viscoelastic flow with large data. J. Differential Equations, 249(2010), no.5, 1179-1198.

[14] X. P. Hu and D. H. Wang. Global existence for the multi-dimensional compressible viscoelastic flows. J. Differential Equations, 250(2011), no.2, 1200-1231.

[15] X. P. Hu and D. H. Wang. Strong solutions to the three-dimensional compressible viscoelastic fluids. J. Differential Equations, 252(2012), no.6, 4027-4067.

[16] X. P. Hu and D. H. Wang. The initial-boundary value problem for the compressible viscoelastic flows. Discrete Contin. Dyn. Syst., 35(2015), no.3, 917-934.

[17] X. P. Hu and G. C. Wu. Global existence and optimal decay rates for three-dimensional compressible viscoelastic flows. SIAM J. Math. Anal., 45(2013), no.5, 2815-2833.

[18] F. Irgens. Rheology and Non-Newtonian Fluids. Springer, Cham, 2014.

[19] F. Jiang, S. Jiang, and G. C. Wu. On stabilizing effect of elasticity in the Rayleigh-Taylor problem of stratified viscoelastic fluids. J. Funct. Anal., 272(2017), no.9, 3763-3824.

[20] F. Jiang, G. C. Wu, and X. Zhong. On exponential stability of gravity driven viscoelastic flows. J. Differential Equations, 260(2016), no.10, 7498-7534.

[21] D. D. Joseph. Fluid Dynamics of Viscoelastic Liquids, volume 84 of Applied Mathematical Sciences. Springer-Verlag, New York, 1990.

[22] Y.KageiandS.Kawashima. Local solvability of an initial boundary value problem for a quasilinear hyperbolic-parabolic system. J. Hyperbolic Differ. Equ., 3(2006), no.2, 195-232.

[23] R. G. Larson. The Structure and Rheology of Complex Fluids. Oxford University Press, New York, 1999.

[24] Z. Lei. On 2D viscoelasticity with small strain. Arch. Ration. Mech. Anal., 198(2010), no.1, 13-37.

[25] Z. Lei, C. Liu, and Y. Zhou. Global existence for a 2D incompressible viscoelastic model with small strain. Commun. Math. Sci., 5(2007), no.3, 595-616.

[26] Z. Lei, C. Liu, and Y. Zhou. Global solutions for incompressible viscoelastic fluids. Arch. Ration. Mech. Anal., 188(2008), no.3, 371-398.

[27] Z. Lei and Y. Zhou. Global existence of classical solutions for the two-dimensional Oldroyd model via the incompressible limit. SIAM J. Math. Anal., 37(2005), no.3, 797-814.

[28] H. L. Li, A. Matsumura, and G. J. Zhang. Optimal decay rate of the compressible Navier-Stokes-Poisson system in $\mathbb{R}^{3}$. Arch. Ration. Mech. Anal., 196(2010), no.2, 681-713.

[29] Y. Li, R. Y. Wei, and Z. A. Yao. Optimal decay rates for the compressible viscoelastic flows. J. Math. Phys., 57(2016), no.11, 111506, 8pp.

[30] F. H. Lin, C. Liu, and P. Zhang. On hydrodynamics of viscoelastic fluids. Comm. Pure Appl. Math., 58(2005), no.11, 14371471.

[31] F. H. Lin and P. Zhang. On the initial-boundary value problem of the incompressible viscoelastic fluid system. Comm. Pure Appl. Math., 61(2008), no.4, 539-558.

[32] C. Liu and N. J. Walkington. An Eulerian description of fluids containing visco-elastic particles. Arch. Ration. Mech. Anal., 159(2001), no.3, 229-252.

[33] H. R. Liu and H. Zhong. Global solutions to the initial boundary problem of 3-D compressible Navier-Stokes-Poisson on bounded domains. Z. Angew. Math. Phys., 72(2021), no.2, 78.

[34] A. Matsumura and T. Nishida. The initial value problem for the equations of motion of compressible viscous and heatconductive fluids. Proc. Japan Acad. Ser. A Math. Sci., 55(1979), no.9, 337-342. 
[35] A. Matsumura and T. Nishida. The initial value problem for the equations of motion of viscous and heat-conductive gases. J. Math. Kyoto Univ., 20(1980), no.1, 67-104.

[36] A. Matsumura and T. Nishida. Initial-boundary value problems for the equations of motion of general fluids. In Computing methods in applied sciences and engineering, V (Versailles, 1981), pages 389-406. North-Holland, Amsterdam, 1982.

[37] A. Matsumura and T. Nishida. Initial-boundary value problems for the equations of motion of compressible viscous and heat-conductive fluids. Comm. Math. Phys., 89(1983), no.4, 445-464.

[38] L. Nirenberg. On elliptic partial differential equations. Ann. Scuola Norm. Sup. Pisa, 13(1959), 115-162.

[39] G. Ponce. Global existence of small solutions to a class of nonlinear evolution equations. Nonlinear Anal., 9(1985), no.5, 399-418.

[40] J. Z. Qian. Initial boundary value problems for the compressible viscoelastic fluid. J. Differential Equations, 250(2011), no.2, 848-865.

[41] J. Z. Qian and Z. F. Zhang. Global well-posedness for compressible viscoelastic fluids near equilibrium. Arch. Ration. Mech. Anal., 198(2010), no.3, 835-868.

[42] M. Renardy, W. J. Hrusa, and J. A. Nohel. Mathematical Problems in Viscoelasticity, volume 35 of Pitman Monographs and Surveys in Pure and Applied Mathematics. Longman Scientific \& Technical, Harlow; John Wiley \& Sons, Inc., New York, 1987.

[43] M. E. Schonbek. $L^{2}$ decay for weak solutions of the Navier-Stokes equations. Arch. Rational Mech. Anal., 88(1985), no.3, 209-222.

[44] M. E. Schonbek. The Fourier splitting method. In Advances in geometric analysis and continuum mechanics (Stanford, CA, 1993), pages 269-274. Int. Press, Cambridge, MA, 1995.

[45] T. C. Sideris and B. Thomases. Global existence for three-dimensional incompressible isotropic elastodynamics via the incompressible limit. Comm. Pure Appl. Math., 58(2005), no.6, 750-788.

[46] Z. Tan, Y. Wang, and W. P. Wu. Mathematical modeling and qualitative analysis of viscoelastic conductive fluids. Anal. Appl. (Singap.), 18(2020), no.6, 1077-1117.

[47] Z. Tan, T. Yang, H. J. Zhao, and Q. Y. Zou. Global solutions to the one-dimensional compressible Navier-Stokes-Poisson equations with large data. SIAM J. Math. Anal., 45(2013), no.2, 547-571.

[48] R. Temam. Navier-Stokes Equations. Theory and Numerical Analysis. North-Holland Publishing Co., Amsterdam-New YorkOxford, 1977. Studies in Mathematics and its Applications, Vol. 2.

[49] C. Truesdell and W. Noll. The Non-linear Field Theories of Mechanics. Springer-Verlag, Berlin, third edition, 2004. Edited and with a preface by Stuart S. Antman.

[50] W. K. Wang and Z. G. Wu. Pointwise estimates of solution for the Navier-Stokes-Poisson equations in multi-dimensions. J. Differential Equations, 248(2010), no.7, 1617-1636.

[51] Y. J. Wang. Decay of the Navier-Stokes-Poisson equations. J. Differential Equations, 253(2012), no.1, 273-297.

[52] Y. Z. Wang and K. Y. Wang. Asymptotic behavior of classical solutions to the compressible Navier-Stokes-Poisson equations in three and higher dimensions. J. Differential Equations, 259(2015), no.1, 25-47.

[53] G. C. Wu, Z. S. Gao, and Z. Tan. Time decay rates for the compressible viscoelastic flows. J. Math. Anal. Appl., 452(2017), no.2, 990-1004.

[54] G. J. Zhang, H. L. Li, and C. J. Zhu. Optimal decay rate of the non-isentropic compressible Navier-Stokes-Poisson system in $\mathbb{R}^{3}$. J. Differential Equations, 250(2011), no.2, 866-891.

[55] T. Zhang and D. Y. Fang. Global existence of strong solution for equations related to the incompressible viscoelastic fluids in the critical $L^{p}$ framework. SIAM J. Math. Anal., 44(2012), no.4, 2266-2288. 